\title{
Wind Energy Bibliography
}

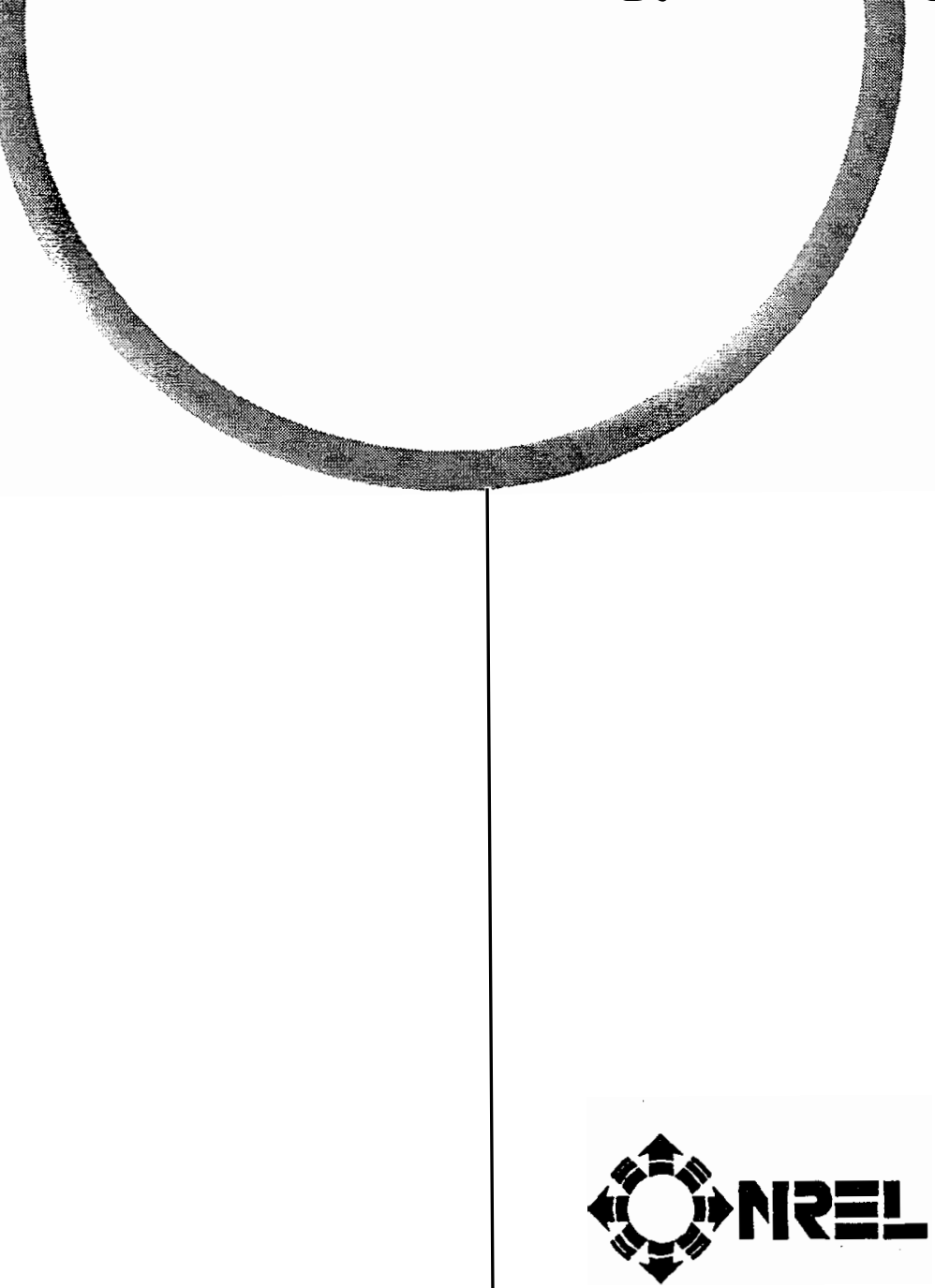

National Renewable Energy Laboratory 1617 Cole Boulevard

Golden, Colorado 80401-3393

A national laboratory of the U.S. Department of Energy Managed by Midwest Research Institute

for the U.S. Department of Energy under contract No. DE-AC36-83CH10093

Prepared under Task No. WE517110

May 1995 


\section{NOTICE}

This report was prepared as an account of work sponsored by an agency of the United States government. Neither the United States government nor any agency thereof, nor any of their employees, makes any warranty, express or implied, or assumes any legal liability or responsibility for the accuracy, completeness, or usefulness of any information, apparatus, product, or process disclosed, or represents that its use would not infringe privately owned rights. Reference herein to any specific commercial product, process, or service by trade name, trademark, manufacturer, or otherwise does not necessarily constitute or imply its endorsement, recommendation, or favoring by the United States government or any agency thereof. The views and opinions of authors expressed herein do not necessarily state or reflect those of the United States government or any agency thereof.

Available to DOE and DOE contractors from:

Office of Scientific and Technical Information (OSTI)

P.O. Box 62

Oak Ridge, TN 37831

Prices available by calling (423) 576-8401

Available to the public from:

National Technical Information Service (NTIS)

U.S. Department of Commerce

5285 Port Royal Road

Springfield, VA 22161

(703) $487-4650$ 


\section{Preface}

This bibliography is designed to help the reader search for information on wind energy. The bibliography is intended to help several audiences, including engineers and scientists who may be unfamiliar with a particular aspect of wind energy, university researchers who are interested in this field, manufacturers who want to learn more about specific wind topics, and librarians who provide information to their clients.

Topics covered range from the history of wind energy use to advanced wind turbine design. In addition to wind turbine development, design, and applications, references for wind energy economics, the wind energy resource, and environmental and institutional issues related to wind energy are also included.

Please note that the references appearing throughout this bibliography do not represent all available material on a specific topic. The inclusion of references in the bibliography is based on several factors, including relevancy to the particular topic, frequency of citation in the professional literature, recommendations from research staff, currency, and availability.

The publisher or sponsoring organization listed in each citation is often the primary distributor of the documents. The appropriate stock number or order number is included in the citation. Requests for government documents can be sent to

Superintendent of Documents

U.S. Government Printing Office

Washington, DC 20402

(202) 783-3238

Requests for U.S. Department of Energy (DOE) or DOE contractor reports can be sent to

National Technical Information Service

Energy Distribution Center

P.O. Box 1300

Oak Ridge, TN 37831

(615) 576-1301 


\section{Table of Contents}

Preface $\ldots \ldots \ldots \ldots \ldots \ldots \ldots \ldots \ldots \ldots \ldots \ldots \ldots \ldots \ldots \ldots \ldots \ldots \ldots \ldots \ldots$ iii

Chapter 1 Introduction $\ldots \ldots \ldots \ldots \ldots \ldots \ldots \ldots \ldots \ldots \ldots \ldots \ldots$

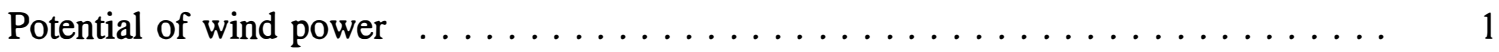

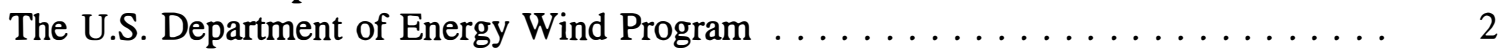

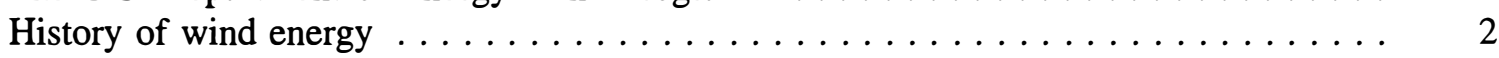

How wind machines capture the energy of the wind $\ldots \ldots \ldots \ldots \ldots \ldots \ldots$

Chapter 2 Economics of Using Wind Energy $\ldots \ldots \ldots \ldots \ldots \ldots \ldots \ldots$

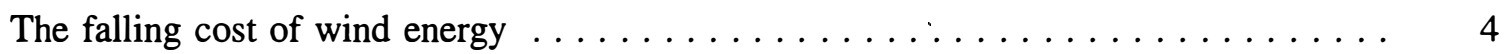

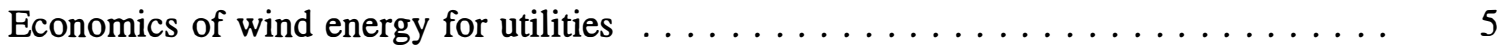

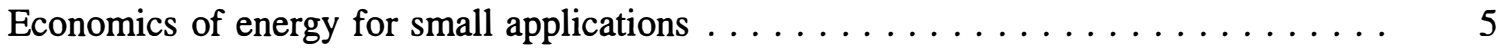

Chapter 3 Wind Energy Resource $\ldots \ldots \ldots \ldots \ldots \ldots \ldots \ldots \ldots \ldots \ldots \ldots$

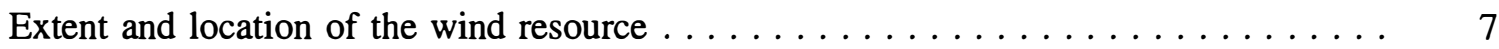

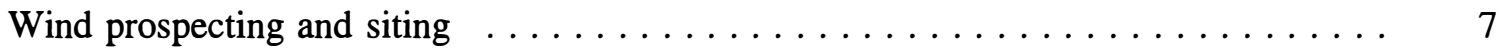

Chapter 4 Wind Turbine Design, Development, and Testing $\ldots \ldots \ldots \ldots \ldots$

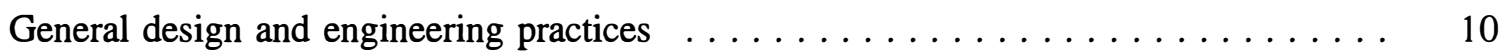

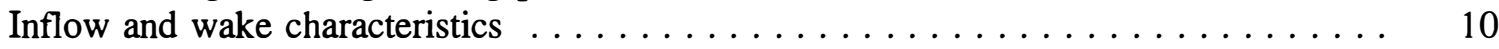

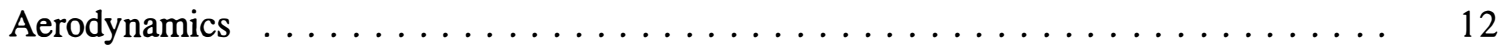

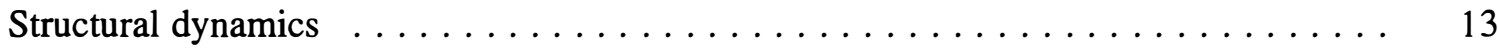

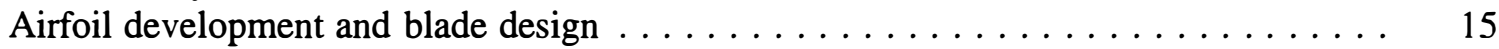

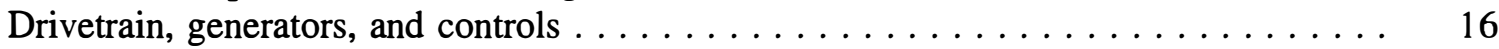

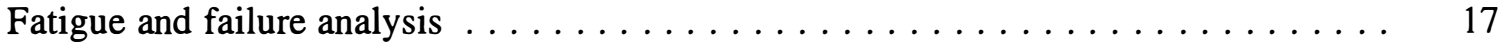

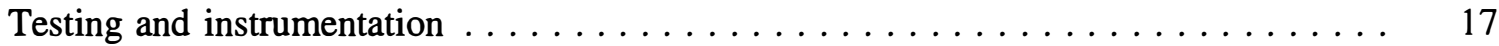

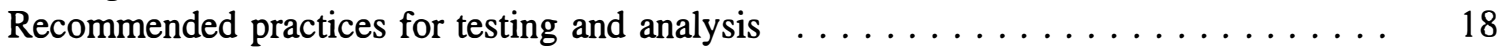

Chapter 5 Applications $\ldots \ldots \ldots \ldots \ldots \ldots \ldots \ldots \ldots \ldots \ldots \ldots \ldots \ldots$

General ......................................... 19

Operation, maintenance, and performance of large wind power plants . . . . . . . 20

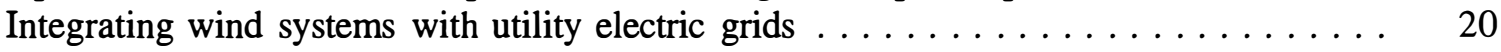

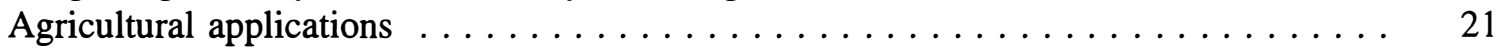

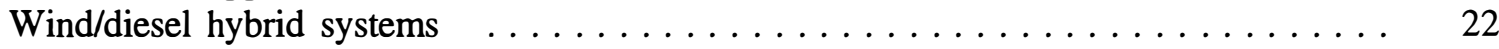

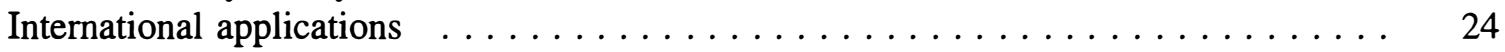

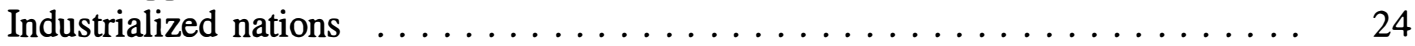

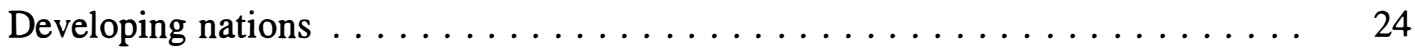




\section{Table of Contents (Continued)}

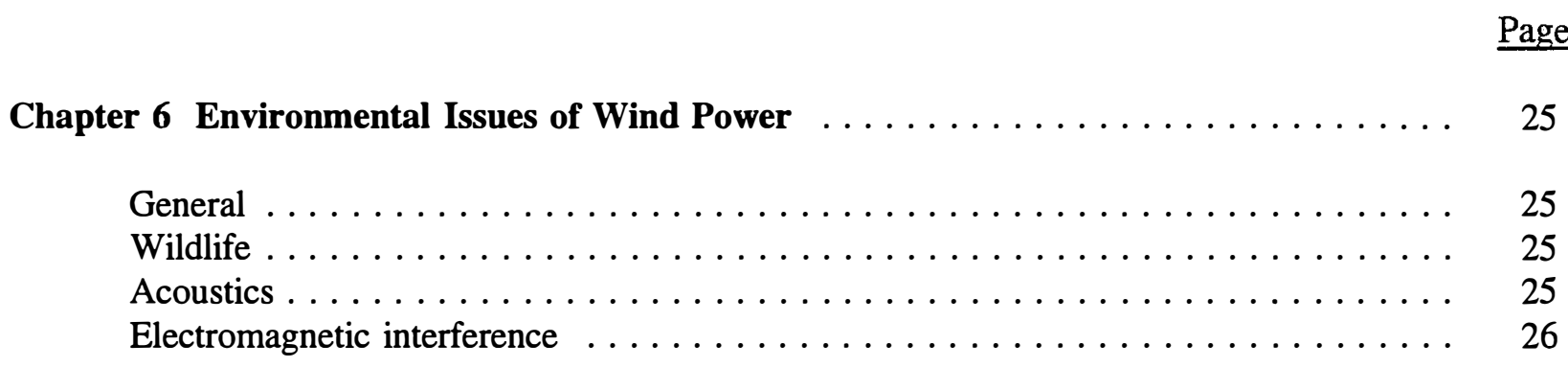

Chapter 7 Institutional Issues and Standards $\ldots \ldots \ldots \ldots \ldots \ldots \ldots \ldots$

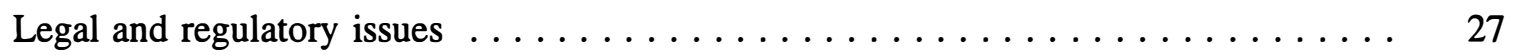

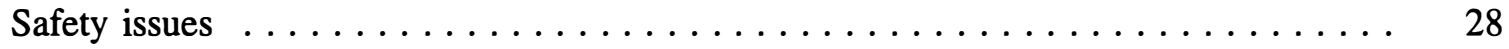

Standards and recommended practices $\ldots \ldots \ldots \ldots \ldots \ldots \ldots \ldots \ldots \ldots \ldots \ldots$

Chapter 8 Wind Energy Systems Development $\ldots \ldots \ldots \ldots \ldots \ldots \ldots \ldots \ldots \ldots$

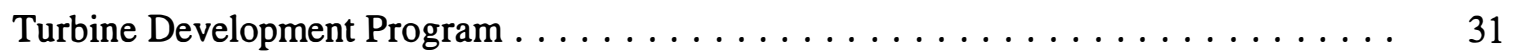

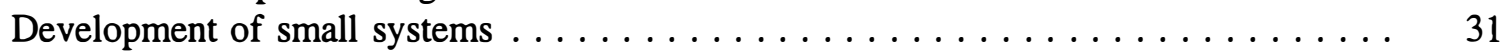

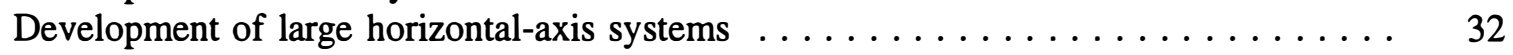

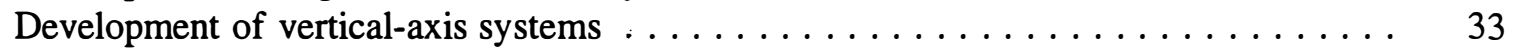

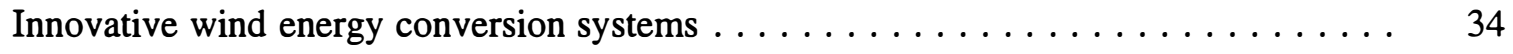

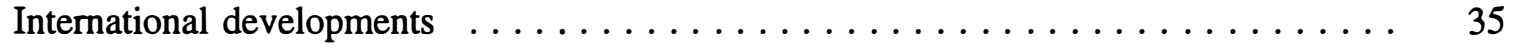

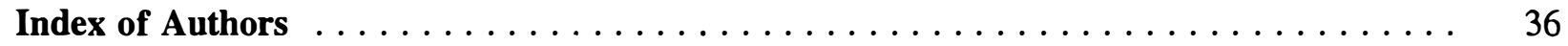

Index of Companies and Organizations $\ldots \ldots \ldots \ldots \ldots \ldots \ldots \ldots \ldots$ 


\section{Chapter 1 Introduction}

\section{Potential of wind power}

National Renewable Energy Laboratory. (1995). International Energy Agency (IEA) Wind Energy Annual Report 1994. NREL/SP-440-7810. Golden, CO: National Renewable Energy Laboratory.

Brower, M.S.; Tennis, M.W.; Denzler, E.W.; Kaplan, M.M. (1993). Powering the Midwest: Renewable Electricity for the Economy and the Environment. Cambridge, MA: Union of Concerned Scientists; 188 pp.

Cavallo, A.J.; Hock, S.M.; Smith, D.R. (1993). "Wind Energy: Technology and Economics." Chapter 3 in Renewable Energy: Sources for Fuels and Electricity. Edited by T.B. Johansson, H. Kelly, A.K.N. Reddy, and R.H. Williams. Washington, DC: Island Press; pp. 121-156.

Van Wijk, A.J.M.; Coelingh, J.P. (1993). Wind Power Potential in the OECD Countries. 93091. Utrecht, The Netherlands: Utrecht University; 35 pp.

Dodge, D.M. (June 1993). "Wind Power Rising." The World \& I. Washington, DC: The Washington Times Corporation; pp. 187-193.

Lamarre, L. (December 1992). "Building Interest in Wind." EPRI Journal. Palo Alto, CA: Electric Power Research Institute; 12 pp.

Hock, S.M.; Thresher, R.W.; Williams, T. (1992). "The Future of Utility-Scale Wind Power." Chapter 9 in Advances in Solar Energy: An Annual Review of Research and Development. Edited by K.W. Boer. Vol. 7. Boulder, CO: American Solar Energy Society; pp. 309-371.

California Energy Commission. (1992). 1992 Energy Technology Status Report. P500-92-007E. Sacramento, CA: California Energy Commission; 82 pp.

American Wind Energy Association. (1992). Wind Energy for Sustainable Development. Washington, DC: American Wind Energy Association; 22 pp.

The Potential of Renewable Energy, An Interlaboratory White Paper; Appendix F. (March 1990). Prepared for the Office of Policy, Planning, and Analysis, U.S. Department of Energy, in support of the National Energy Strategy. SERITP-260-3674. Available from the National Renewable Energy Laboratory, Golden, CO; 11 pp. (NTIS no. DE90000322).

Swisher, R. (American Wind Energy Association); Ancona, D.F. (U.S. Department of Energy); Edworthy, J. (Canadian Wind Energy Association). (September 1990). "Wind Energy Developments in the Americas." European Community Wind Energy Conference and Exhibition; September 10-14, 1990; Madrid, Spain. Bedford, England: H.S. Stephens \& Associates; pp. 43-48. 


\section{The U.S. Department of Energy Wind Program}

Wind Energy Program Overview, Fiscal Year 1994. (March 1995). DOE/GO-10095-071. Golden, CO: National Renewable Energy Laboratory. (NTIS no. DE95000288).

Wind Energy Program Overview, Fiscal Year 1993. (May 1994). DOE/CH10093-279. Golden, CO: National Renewable Energy Laboratory. (NTIS no. DE94000285).

Wind Energy Program Overview, Fiscal Year 1992. (April 1993). DOE/CH10093-191. Golden, CO: National Renewable Energy Laboratory. (NTIS no. DE93000056).

Bollmeier, W.S., II; Dodge, D.M. (May 1992). Cooperative Field Test Program for Wind Systems. NREL/TP-253-4252. Golden, CO: National Renewable Energy Laboratory; 38 pp. (NTIS no. DE92001209).

A Government-Industry Partnership Experience: The Cooperative Field Test Program in Wind Research. (May 1991). 6 pp. Available from American Wind Energy Association, Washington, DC.

\section{History of wind energy}

Shepherd, D.G. (Comell University). (December 1990). Historical Development of the Windmill. (DOE/NASA-5266-2). Washington, DC: U.S. Department of Energy; 45 pp. (NTIS no. DE91006447/XAB).

Baker, T.L. (1985). A Field Guide to American Windmills. Norman, OK: University of Oklahoma Press; 516 pp.

Johnson, G.L. (1985). Wind Energy Systems. New York, NY: Prentice Hall.

Five Year Research Plan 1985-1990, Wind Energy Technology: Generating Power from the Wind. (January 1985). DOE/CE-T11. Washington, DC: U.S. Department of Energy; 32 pp. (NTIS no. DE85008427/XAB).

Fleming, P.D.; Probert, S.D. (April 1982). "Sail-Type Wind-Turbines: A Review and Annotated Bibliography." Applied Energy (10:4); pp. 273-286.

Koeppl, G.W. (1982). Putnam's Power From the Wind. 2nd edition. New York, NY: Van Nostrand Reinhold Company, Inc.; 470 pp.

Golding, E.W. (1976). The Generation of Electricity by Wind Power. New York, NY: John Wiley \& Sons; $332 \mathrm{pp}$.

Torrey, V. (1976). Wind Catchers: American Windmills of Yesterday and Tomorrow. Brattleboro, VT: The Stephen Greene Press; 226 pp. 


\section{How wind machines capture the energy of the wind}

Spera, D.A. (May 1994). Wind Turbine Technology: Fundamental Concepts of Wind Turbine Engineering. No. 100368. Fairfield, NJ: American Society of Mechanical Engineers; 700 pp.

Nelson, V.; Gilmore, E.H.; Starcher, K. (1993). Introduction to Wind Energy. 93-3. Canyon, TX: Alternative Energy Institute; 28 pp.

Introduction to Small Wind Systems. (1993). Washington, DC: American Wind Energy Association; $22 \mathrm{pp}$.

Gipe, P. (1993). Wind Power for Home \& Business. Post Mills, VT: Chelsea Green Publishing Company; 413 pp.

Ancona, D.F. (1989). "Power Generation, Wind, Ocean." Wilk's Encyclopedia of Architecture: Design, Engineering, and Construction. Vol. 4. New York, NY: John Wiley \& Sons; pp. 32-39.

Dodge, D.M.; Thresher, R.W. (1989). "Wind Technology Today." Advances in Solar Energy, An Annual Review of Research and Development. Vol. 5. New York, NY: Plenum Press.

Eggleston, D.M.; Stoddard, F.S. (1987). Wind Turbine Engineering Design. New York, NY: Van Nostrand Reinhold Company, Inc.; 352 pp.

Moretti, P.M.; Divone, L.V. (June 1986). "Modern Windmills." Scientific American (254:6); pp. 110-118.

Le Gourieres, D. (1982). Wind Power Plants, Theory and Design. New York, NY: Pergamon Press.

Park, J. (1981). The Wind Power Book. Palo Alto, CA: Cheshire Books; 253 pp.

Eldridge, F.R. (1980). Wind Machines. 2nd edition. New York, NY: Van Nostrand Reinhold Company, Inc.; $214 \mathrm{pp}$.

Park, J.; Schwind, D. (September 1978). Wind Power for Farms, Houses, and Small Industry. RFP-2841/1270/78/4. Mountain View, CA: Nielsen Engineering and Research, Inc.; 229 pp. 


\section{Chapter 2 Economics of Using Wind Energy}

\section{The falling cost of wind energy}

Milligan, M.R.; Miller, A.H. (July 1993). The Value of Windpower: An Investigation Using a Qualified Production Cost Model. NREL/TP-441-5730. Golden, CO: National Renewable Energy Laboratory; $8 \mathrm{pp}$.

Economic Lessons From a Decade of Experience. (1993). Produced under the auspices of the Utility Wind Interest Group; published by the Electric Power Research Institute; 4 pp. Available from the National Renewable Energy Laboratory, Golden, CO.

Swift, A. (University of Texas); Thresher, R.W.; Hock, S.M. (National Renewable Energy Laboratory). (1992). "Performance and Cost Projections for Advanced Wind Turbines." Windpower '92 Conference; October 19-23, 1992; Seattle, Washington. Washington, DC: American Wind Energy Association; pp. $431-447$.

Karas, K.C. (1992). "Wind Energy: What Does it Really Cost?" Windpower '92 Conference; October 19-23, 1992; Seattle, Washington. Arlington, VA: American Wind Energy Association. pp. 157-166. Also NREL/TP-441-5245. Golden, CO: National Renewable Energy Laboratory; 10 pp. (NTIS no. DE93000044).

Dodd, H.M.; Hock, S.M.; Thresher, R.W. (1990). "The Sensitivity of Wind Technology Utilization to Cost and Market Parameters." Windpower '90 Conference; September 25, 1990; Washington, DC. Arlington, VA: American Wind Energy Association. Also SERITP-257-3986. Golden, CO: National Renewable Energy Laboratory; 6 pp. (NTIS no. DE91002122).

Merrill, O.; Schweizer, T.; Chernoff, H.; Conopask, H.; Knowles, R.; Moyle, R.A. (August 1985). Early Market Potential for Utility Applications of Wind Turbines. EPRI-AP-4077. Palo Alto, CA: Electric Power Research Institute; 200 pp.

Recommended Practices for Wind Turbine Testing: 2. Estimation of Cost of Energy from Wind Energy Conversion Systems; 1. Edition 1983. (1983). Edited by J. Nitteberg, A.A. deBoer, and P.B. Simpson. 23 pp. Submitted to the Executive Committee of the International Energy Agency Program for Research and Development on Wind Energy Conversion Systems. (NTIS no. DE84901132).

Moyle, R.A.; Chernoff, H.; Schweizer, T.C.; Patton, J.B. (November 1982). Assessment of Distributed Solar Power Systems: Issues and Impacts. EPRI-AP-2636. Palo Alto, CA: Electric Power Research Institute; $184 \mathrm{pp}$.

Britt, J.F.; Davey, H.L.; McCarroll, D.A.; Wysocki, K.J. (December 1981). Small Wind Turbine Production Evaluation and Cost Analysis. SERITR-09049-1. Golden, CO: Solar Energy Research Institute; 2 Vols. 


\section{Economics of wind energy for utilities}

TAG TM Technical Assessment Guide; Volume 1: Electricity Supply-1993 (Revision 7). (June 1993). Technology and Fuels Assessment Department, Electric Power Research Institute. TR-102276-V1R7. Palo Alto, CA: Electric Power Research Institute.

Cohen, J.M.; Schweizer, T.C. (Science Applications International Corporation); Hock, S.M. (National Renewable Energy Laboratory); Cadogan, J.B. (DOE). (1992). A Methodology for Computing Wind Turbine Cost of Electricity Using Utility Economic Assumptions (Revised).

Bergey, K.H. (Bergey Windpower Company, Inc.). (1991). "Windfarm Strategies: The Economies of Scale Revisited." Windpower '91 Conference; September 24-27, 1991; Palm Springs, California. Washington, DC: American Wind Energy Association; pp. 72-81.

Hock, S.M.; Thresher, R.W. (NREL); Cohen, J.M. (Science Applications International Corporation). (1990). "Performance and Cost Projections for Advanced Wind Turbines." American Society of Mechanical Engineers, Winter Annual Meeting; November 25, 1990; Dallas, Texas. Also SERITP-257-3795. Golden, CO: Solar Energy Research Institute; 7 pp. (NTIS no. DE90000370).

Merrill, O.; Schweizer, T.; Chernoff, H.; Conopask, H.; Knowles, R.; Nagle, R. (Science Applications International Corporation). (August 1985). Early Market Potential for Utility Applications of Wind Turbines. EPRI-AP-4077. Palo Alto, CA: Electric Power Research Institute; 200 pp.

Flaim, T.; Hock, S.M. (May 1984). Wind Energy Systems for Electric Utilities: A Synthesis of Value Studies. SERI/TR-211-2318. Golden, CO: Solar Energy Research Institute; 112 pp.

Sullivan, R.L.; Flaim, T.; Percival, D. (March 1982). WECS Value Analysis: A Comparative Assessment of Four Methods. SERI/TR-211-1563. Golden, CO: Solar Energy Research Institute; 51 pp.

Percival, D.; Harper, J. (January 1982). Electric Utility Value Determination for Wind Energy. Vol. I: A Methodology. SERI/TR-732-604R-V.1. 94 pp. (NTIS no. DE82010771). Vol. II: A User's Guide. SERI/TR-732-604R-V.2. 121 pp. (NTIS no. DE82010926). Golden, CO: Solar Energy Research Institute.

\section{Economics of wind energy for small applications}

Evaluating Residential Wind System Economics. (1994). Washington, DC: American Wind Energy Association; 20 pp.

Wyatt, A. (Research Triangle Institute). (1992). Wind Electric Pumping Systems: Sizing and Cost Estimation. Washington, DC: American Wind Energy Association; 39 pp.

Moment, R.L. (February 1983). Performance and Size Estimating for Wind Systems. RFP-3586. Golden, CO: Rockwell International Corporation; 69 pp. (NTIS no. DE84006980).

JBF Scientific Corporation. (June 1981). Small Wind Systems Application Analysis. RFP-3147/2. Golden, CO: Rockwell International Corporation; 217 pp. (NTIS no. DE82003748). 
Stafford, R.W.; Greeb, F.J.; Smith, M.F.; Des Chenes, C.; Weaver, N.L. (January 1981). Economic Analysis of Wind-Powered Farmhouse and Farm Building Heating Systems; Final Report. DOE/SEA-3408-20691/81/1. Washington, DC: U.S. Department of Energy; 360 pp. Available from NTIS.

Garling, W.S.; Haufer, M.R.; Merchang-Geuder, L; Welch, M. (March 1980). Economic Analysis of Wind-Powered Refrigeration Cooling/Water Heating Systems in Food Processing. DOE/SEA-1109-20401/81/1. Washington, DC: U.S. Department of Energy; 150 pp. Available from NTIS. 


\section{Chapter 3 Wind Energy Resource}

\section{Extent and location of the wind resource}

Pacific Northwest Laboratory. (1993). Shaded Relief Elevation Maps for Wind Prospectors. Washington, DC: American Wind Energy Association.

Elliott, D.L.; Schwartz, M.N. (September 1993). Wind Energy Potential in the United States. PNL-SA-23109. Richland, WA: Pacific Northwest Laboratory; 10 pp. (NTIS no. DE94001667).

Schwartz, M.N.; Elliott, D.L.; Gower, G.L. (1992). "Gridded State Maps of Wind Electric Potential." Windpower '92 Conference; October 19-23, 1992; Seattle, Washington. Washington, DC: American Wind Energy Association; pp. 50-58.

America Takes Stock of a Vast Energy Resource. (1992). Produced under the auspices of the Utility Wind Interest Group; published by the Electric Power Research Institute; $10 \mathrm{pp}$. Available from the National Renewable Energy Laboratory, Golden, CO.

Elliott, D.L.; Gower, G.L.; Wendell, L.L. (August 1991). An Assessment of the Available Windy Land Area and Wind Energy Potential in the Contiguous United States. PNL-7789. Richland, WA: Pacific Northwest Laboratory; 92 pp. (NTIS no. DE91018887).

Elliott, D.L. (October 1987). Caribbean and Central America Wind Energy Assessment. PNL-SA-15102. Richland, WA: Pacific Northwest Laboratory.

Elliott, D.L.; Holladay, C.G.; Barchet, W.R.; Foote, H.P.; Sandusky, W.F. (March 1987). Wind Energy Resource Atlas of the United States. DOE/CH10093-4. Richland, WA: Pacific Northwest Laboratory; 210 pp. (NTIS no. DE86004442).

California Wind Atlas. (April 1985). Sacramento, CA: California Energy Commission; 210 pp. Available from California Energy Commission, Publication Unit, 1516 Ninth Street, MS-15, Sacramento, CA 95814.

Changery, M.J. (December 1978). National Wind Data Index. HCO/T1041-01. Asheville, NC: National Climatic Data Center; 245 pp.

Changery, M.J.; Hodge, W.T.; Ramsdell, J.V. (September 1977). Index-Summarized Wind Data. BNWL-2220 WIND 11. Richland, WA: Pacific Northwest Laboratory; $151 \mathrm{pp}$.

\section{Wind prospecting and siting}

Recommended Practice for the Siting of Wind Energy Conversion Systems. (1993). AWEA Standard: AWEA 8.2-1993. Washington, DC: American Wind Energy Association.

McCarthy, E.F. (Kenetech/U.S. Windpower, Inc.); Meroney, R.N.; Neff, D.E. (Colorado State University). (1993). "Elevation and Vegetation Considerations on Wind Power Availability." Windpower '93 
Conference; July 12-16, 1993; San Francisco, California. Washington, DC: American Wind Energy Association; p. 213.

Wendell, L.L.; Gower, G.L.; Birn, M.B. (July 1993). "Applicability of Digital Terrain Analyses to Wind Energy Prospecting and Siting." Windpower '93 Conference; July 12-16, 1993; San Francisco, California. Washington, DC: American Wind Energy Association.

Understanding Your Wind Resource. (1994). Washington, DC: American Wind Energy Association; $12 \mathrm{pp}$.

Council, C. (Western Area Power Administration); Quinlan, P.; Garrick, J. (NEOS Corporation). (1991). "Overview of Western Area Power Administration's Wind Prospecting Program." Windpower '91 Conference; September 24-27, 1991; Palm Springs, California. Washington, DC: American Wind Energy Association; pp. 219-225.

Wade, J.E.; Baker, R.W.; Walker, S.N. (Oregon State University). (February 1990). Wind Forecasting for Utility Operations. DOE/BP/63406-9. Washington, DC: U.S. Department of Energy; 62 pp. (NTIS no. DE90009466).

Walker, S.N. (Pacific Wind Energy). (1988). Local Wind Measurements for Micrositing. SERI/STR-217-3320. Golden, CO: Solar Energy Research Institute.

Standard Procedures for Meteorological Measurements at a Potential Wind Turbine Site. (1986). AWEA Standard: AWEA 8.1-1986. Alexandria, VA: American Wind Energy Association; 18 pp.

Pennell, W.T. (January 1983). Siting Guidelines for Utility Application of Wind Turbines. EPRI-AP-2795. Palo Alto, CA: Electric Power Research Institute; 266 pp.

Wade, J.E.; Maule, P.A.; Bodvarsson, G.; Rosenfeld, C.L.; Woolley, S.G.; McClenahan, M.R. (February 1983). Remote Sensing for Wind Power Potential, A Prospector's Handbook. DOE/ET/20316-81-1. Richland, WA: Pacific Northwest Laboratory; 308 pp.

Pennell, W.T.; Miller, A.H. (1981). Procedures for Modeling Wind Turbine Performance from Site Wind Data. 82-Pet-7. New York, NY: The American Society of Mechanical Engineers; 10 pp. Available from Engineering Societies Library, 345 E. 47th Street, New York, NY 10017.

Wade, J.E.; Hewson, E.W. (October 1981). A Guide to Biological Wind Prospecting. DOE/ET/20316-80-2. Corvallis, OR: Oregon State University; 112 pp.

Frost, W.; Shieh, C.F. (December 1981). Guidelines for Siting WECS Relative to Small-Scale Terrain Features. DOE/ET/20242-78/1. Tullahoma, TN: FWG Associates, Inc.; 196 pp.

Meteorological Aspects of the Utilization of Wind as an Energy Source. (1981). Technical Note 175. Geneva, Switzerland: World Meteorological Organization; 180 pp.

Hiester, T.R.; Pennell, W.T. (January 1981). The Meteorological Aspects of Siting Large Wind Turbines. PNL-2522. Richland, WA: Pacific Northwest Laboratory; 512 pp.

Wegley, H.L.; Orgill, M.M.; Drake, R.L. (March 1980). A Siting Handbook for Small Wind Energy Systems. PNL-2521 Rev. 1. Richland, WA: Pacific Northwest Laboratory; 132 pp. 
Corotis, R.B. (May 1980). Application of Statistical Techniques to Wind Characteristics at Potential Wind Energy Conversion Sites. DOE/ET/20283-2. Evanston, IL: Northwestern University; 122 pp.

Proceedings of the Conference and Workshop on Wind Energy Characteristics and Wind Energy Siting; June 19-21, 1979; Portland, Oregon. (1979). PNL-3214. Richland, WA: Pacific Northwest Laboratory; $471 \mathrm{pp}$.

Marrs, R.W.; Gaylord, D.R. (April 1979). A Guide to the Interpretation of Windflow Characteristics from Eolian Landforms. RLO-2343-79/2. Laramie, WY: University of Wyoming; 39 pp. 


\section{Chapter 4 Wind Turbine Design, Development, and Testing}

\section{General design and engineering practices}

Spera, D.A. (May 1994). Wind Turbine Technology: Fundamental Concepts of Wind Turbine Engineering. No. 100368. Fairfield, NJ: American Society of Mechanical Engineers; 700 pp.

Araj, K.J.; Fisher, T.A.; Kronenburg, J.C. (National Research Council, Washington, DC). (1991). Assessment of Research Needs for Wind Turbine Rotor Materials Technology. DOE/AL/58181-T1. Washington, DC: U.S. Department of Energy; 108 pp. (NTIS no. DE92018563).

Design Criteria Recommended Practices: Wind Energy Conversion Systems. (1988). AWEA Standard: AWEA 3.1-1988. Arlington, VA: American Wind Energy Association; 25 pp.

Eggleston, D.M.; Stoddard, F.S. (1987). Wind Turbine Engineering Design. New York, NY: Van Nostrand Reinhold Company, Inc.; 352 pp.

Miller, A.H.; Dugundji, J.; Martinez-Sanchez, M.; Gohard, J.; Chung, S.; Wendell, J. (September 1978). Wind Energy Conversion, Volume 1: Methods for Design Analysis of Horizontal Axis Wind Turbines. COO-4131-Tq (Vol. I). Washington, DC: U.S. Department of Energy; 253 pp.

\section{Inflow and wake characteristics}

Wendell, L.L; Barnard, J.C.; Morris, V.R.; Gower, G.L. (1993). "Turbulence Characteristics in Complex Terrain." Wind Energy 1993. SED-Vol. 14, Wind Energy. New York, NY: The American Society of Mechanical Engineers.

Kelley, D.N. (November 1992). Full Vector (3-D) Inflow Simulation in Natural and Wind Farm Environments Using an Expanded Version of the SNLWIND (Veers) Turbulence Code. NREL/TP-4425225. Golden, CO: National Renewable Energy Laboratory; 8 pp.

NREL/TP-442-5225. Golden, CO: National Renewable Energy Laboratory; 8 pp.

Morris, V.R.; Barnard, J.C.; Wendell, L.L.; Tomich, S.D. (October 1992). "Comparison of Anemometers for Turbulence Characterization." Windpower'92 Conference; October 19-23, 1992; Seattle, Washington. Washington, DC: American Wind Energy Association; 9 pp. (NTIS no. DE93004336).

Kelley, N.D.; Wright, A.D. (October 1991). A Comparison of Predicted and Observed Turbulent Wind Fields Present in Natural and Internal Wind Park Environments. NREL/TP-257-4508. Golden, CO: National Renewable Energy Laboratory; 9 pp.

Elliott, D.L. (September 1991). "Status of Wake and Array Loss Research." Windpower '91 Conference; September 24-27, 1991; Palm Springs, California. Washington, DC: American Wind Energy Association. 
Barnard, J.C. (June 1990). An Evaluation of Three Models Designed for Siting Wind Turbines in Areas of Complex Terrain. PNL-7357. Richland, WA: Pacific Northwest Laboratory; 50 pp. (NTIS no. DE90013625).

Nierenberg, R. (Altamont Energy Corporation). (1990). Wake Deficit Measurements on the Jess and Souza Ranches, Altamont Pass. SERI/STR-257-3455. Golden, CO: Solar Energy Research Institute.

Connell, J.R.; Morris, V.R. (1990). "Turbulent Wind at the Equatorial Segment of an Operating Darrieus Wind Turbine Blade." Ninth ASME Wind Energy Symposium. SED-Vol. 9. New York, NY: American Society of Mechanical Engineers.

Elliott, D.L.; Barnard, J.C. (November 1990). "Effects of Trees on Wind Flow Variability and Turbulence." Journal of Solar Energy Engineering; Vol. 112. New York, NY: American Society of Mechanical Engineers.

Elliott, D.L.; Cadogan, J.B. (1990). "Effects of Wind Shear and Turbulence on Wind Turbine Power Curves." European Community Wind Energy Conference and Exhibition; September 10-14, 1990; Madrid, Spain. Bedford, England: H.S. Stephens \& Associates.

Kelley, N.D. (September 1989). An Initial Look at the Dynamics of the Microscale Flow Field Within a Large Windfarm in Response to Variations in the Natural Inflow. SERI/TP-257-3591. Golden, CO: National Renewable Energy Laboratory; 5 pp.

Connell, J.R.; Powell, D.C. (August 1989). User's Guide for Personal Computer Model of Turbulence at a Wind Turbine Rotor. PNL-6959. Richland, WA: Pacific Northwest Laboratory.

Nierenberg, R. (Altamont Energy Corporation). (1989). Free-Flow Variability on the Jess and Souza Ranches, Altamont Pass. SERI/STR-217-3404. Golden, CO: Solar Energy Research Institute.

Elliott, D.L.; Buck, J.W.; Barnard, J.C. (April 1988). An Examination of Wake Effects and Power Production for a Group of Large Wind Turbines. PNL-6528. Richland, WA: Pacific Northwest Laboratory; 58 pp. (NTIS no. DE88009906).

Veers, P.S. (1988). Three-Dimensional Wind Simulation. SAND-88-0152. Albuquerque, NM: Sandia National Laboratories; 36 pp. (NTIS no. DE88009793/XSP).

Connell, J.R. (1988). "A Primer of Turbulence at the Wind Turbine Rotor." Solar Energy (41:3); pp. 281-293. New York, NY: Pergamon Press.

Simon, R.L. (Fayette Manufacturing Corporation). (April 1988). Wake Effects in a Fayette 85-IIS Wind Turbine Array. SERI/STR-217-3186A. Golden, CO: Solar Energy Research Institute; 75 pp.

Liu, H.-T. (FloWind Corporation). (1988). Field Investigation of a Wake Structure Downwind of a VAWT in a Wind Farm Array. SERI/STR-217-3370. Golden, CO: Solar Energy Research Institute.

Barnard, J.C.; Wegley, H.L. (January 1987). A Preliminary Evaluation of the Performance of Wind Tunnel and Numerical Modeling Simulations of the Wind. PNL-6105. Richland, WA: Pacific Northwest Laboratory; 20 pp. (NTIS no. DE87004634). 
Simon, R.L.; Matson, D.F.; Fuchs, J.M. (Fayette Manufacturing Corporation). (1987). Wake Effects in a Fayette 95-IIS Wind Turbine Array. SERI/STR-217-3186. Golden, CO: Solar Energy Research Institute.

Connell, J.R.; George, R.L. (November 1987). "Accurate Correlation of Wind Turbine Response With Wind Speed Using a New Characterization of Turbulent Wind." Journal of Solar Energy Engineering; Vol. 109. New York, NY: American Society of Mechanical Engineers.

Barnard, J.C.; Wegley, H.L.; Hiester, T.R. (September 1985). Improving the Performance of MassConsistent Numerical Models Using Optimization Techniques. PNL-5566. Richland, WA: Pacific Northwest Laboratory; 33 pp. (NTIS no. DE86001224). An article based on this report appeared in the Journal of Climate and Applied Meteorology (26:6); pp. 675-686.

Buck, J.W.; Renne, D.S. (August 1985). Observations of Wake Characteristics at the Goodnoe Hills MOD-2 Array. PNL-5565. Richland, WA: Pacific Northwest Laboratory; $77 \mathrm{pp}$. (NTIS no. DE85018282).

Lissaman, P.B.S.; Gyatt, G.W.; Zalay, A.D. (AeroVironment). (June 1982). Numeric Modeling Sensitivity Analysis of the Performance of Wind Turbine Arrays. PNL-4183. Richland, WA: Pacific Northwest Laboratory; $97 \mathrm{pp}$.

Doran, J.C.; Packard, K.R. (October 1982). Comparison of Model and Observations of the Wake of a MOD-OA Wind Turbine. PNL-4433. Richland, WA: Pacific Northwest Laboratory; 43 pp.

Doran, J.C. (August 1981). Inherent Uncertainties in Meteorological Parameters for Wind Turbine Design. PNL-SA-736. Richland, WA: Pacific Northwest Laboratory; 18 pp.

Frost, W.; Long, B.H.; Turner, R.E. (December 1978). Engineering Handbook on the Atmospheric Environmental Guidelines for Use in Wind Turbine Generator Development. NASA-TP-1359. Huntsville, AL: National Aeronautics and Space Administration, Marshall Space Flight Center; 372 pp.

\section{Aerodynamics}

McNerney, G.M. (U.S. Windpower, Inc.). (1994). "U.S. Windpower 56-100 Stall Operation." Wind Energy 1994. SED-Vol. 15. New York, NY: American Society of Mechanical Engineers; pp. 219-226.

Hansen, A.C.; Butterfield, C.P. (1993). "Aerodynamics of Horizontal-Axis Wind Turbines." Annual Review of Fluid Mechanics. Vol. 25, pp. 115-149.

Butterfield, C.P.; Musial, W.P.; Scott, G.N.; Simms, D.A. (1992). NREL Combined Experiment Final Report-Phase II (draft). NREL/TP-442-4807. Golden, CO: National Renewable Energy Laboratory; 32 pp. (plus appendices).

Butterfield, C.P.; Musial, W.P.; Simms, D.A. (1992). Combined Experiment Phase I: Final Report. NREL/TP-257-4655. Golden, CO: National Renewable Energy Laboratory; 220 pp. (NTIS no. DE93000012).

Barnwell, R.W.; Hussaini, M.Y. (1992). Natural Laminar Flow and Laminar Flow Control. New York, NY: Springer-Verlag; 411 pp. 
Eggleston, D.M.; Starcher, K.L. (November 1990). "A Comparative Study of the Aerodynamics of Several Wind Turbines Using Flow Visualization." Journal of Solar Energy Engineering. Vol. 112. New York, NY: American Society of Mechanical Engineers; pp. 301-309.

Thresher, R.W.; Wright, A.D. (January 1989). Prediction of Stochastic Blade Responses Using Measured Wind-Speed Data as Input to the FLAP Code. SERI/TP-217-3394. Golden, CO: Solar Energy Research Institute.

Thresher, R.W.; Holley, W.E.; Wright, A.D. (January 1989). Prediction of Stochastic Blade Responses Using a Filtered Noise Turbulence Model in the FLAP Code. SERITP-217-3413. Golden, CO: Solar Energy Research Institute.

Selig, M.S.; Donovan, J.F.; Fraser, D.B. (1989). Airfoils at Low Speeds. Soartech 8. Virginia Beach, VA: H.A. Stokely; 398 pp.

Walker, S.N.; Wade, J.E. (Pacific Wind Energy). (1988). Effects of Precipitation on Wind Turbine Performance. SERI/STR-217-3287. Golden, CO: Solar Energy Research Institute.

Stoddard, F.S.; Porter, B.K. (January 1986). Wind Turbine Aerodynamics Research Needs Assessment. DOE/ER/30075-H1. Washington, DC: U.S. Department of Energy; 126 pp. (NTIS no. DE86009189).

Wilson, R.E.; Walker, S.N. (1985). "State-of-the-Art in Wind Turbine Aerodynamics." Windpower '85 Conference; August 27, 1985; San Francisco, California. Alexandria, VA: American Wind Energy Association; pp. 30-38.

Noll, R.B.; Ham, N.D. (Aerospace Systems, Inc.). (February 1983). Dynamic Stall of Small Wind Systems. RFP-3523. Golden, CO: National Renewable Energy Laboratory. (NTIS no. DE84006827).

Horizontal-Axis Wind System Rotor Performance Model Comparison-A Compendium. (February 1983). RFP-3508. Golden, CO: Rocky Flats Wind Energy Research Center; 381 pp. (NTIS no. DE83012265).

Viterna, L.A.; Janetzke, D.C. (September 1982). Theoretical and Experimental Power from Large Horizontal-Axis Wind Turbines. DOE/NASA/20320-41. Cleveland, OH: NASA Lewis Research Center; 18 pp. (NTIS no. DE83001099).

Rohrbach, C.; Wainauski, H.; Worabel, R. (December 1977). Experimental and Analytical Research on the Aerodynamics of Wind Driven Turbines. COO-2615-T2. Windsor Locks, CT: Hamilton Standard; $250 \mathrm{pp}$.

\section{Structural dynamics}

Bywaters, G.L. (Northern Power Systems); Manwell, J.F. (University of Massachusetts). (1994). "Teeter Dynamics in Complex Terrain." Wind Energy 1994. SED-Vol. 15. New York, NY: American Society of Mechanical Engineers; pp. 147-156.

Babcock, B.A.; Conover, K.E. (R. Lynette \& Associates, Inc.). (1994). "Design of Cost-Effective Towers for an Advanced Wind Turbine." Wind Energy 1994. SED-Vol. 15. New York, NY: American Society of Mechanical Engineers; pp. 261-268. 
James, G.H., III; Came, T.G.; Lauffer, J.P. (1993). The Natural Excitation Technique (NExT) for Modal Parameter Extraction from Operating Wind Turbines. SAND-92-1666. Albuquerque, NM: Sandia National Laboratories; 46 pp. (NTIS no. DE93010611).

Wilson, R.E.; Walker, S.N.; Smith, C.E.; Freeman, L.N. (1993). "Advanced Dynamics Code." Wind Energy 1993; January 31-February 4, 1993; Houston, Texas. SED-Vol. 14. New York, NY: American Society of Mechanical Engineers; pp. 119-124.

Hansen, A.C. (1992). Yaw Dynamics of Horizontal Axis Wind Turbines: Final Report. NREL/TP-4424822. Golden, CO: National Renewable Energy Laboratory; 175 pp. (NTIS no. DE92001245).

Wright, A.D.; Buhl, M.L., Jr.; Elliott, A.S. (1992). "Development of a Wind Turbine System's Dynamics Model Using the Automatic Dynamic Analysis of Mechanical Systems (ADAMS) Software." Windpower '92 Conference; October 19-23, 1992; Seattle, Washington. Washington, DC: American Wind Energy Association; pp. 329-338.

Weber, T.L.; Wilson, R.E.; Walker, S.N. (Oregon State University). (June 1991). Analysis of a Teetered, Variable-Speed Rotor. SERITP-267-4366. Golden, CO: Solar Energy Research Institute; 59 pp. (NTIS no. DE91002190).

Wright, A.D.; Butterfield, C.P. (1991). The NREL Teetering Hub Rotor Code: Final Results and Conclusions. NREL/TP-257-4517. Golden, CO: National Renewable Energy Laboratory; 10 pp. (NTIS no. DE92001187).

Coleman, C; McNiff, B.P. (Nothern Power Systems). (1990). Dynamic Response Testing of the North Wind 100 Wind Turbine. SERI/STR-257-3686. Golden, CO: Solar Energy Research Institute.

Snow, A.L.; Heberling, C.F., III; Van Bibber, L.E. (Westinghouse Electric Corporation). (March 1989). The Dynamic Response of a Westinghouse 600-kW Wind Turbine. SERI/STR-217-3405. Golden, CO: Solar Energy Research Institute; 185 pp.

Wehrey, M.; Redmond, I.; Anderson, C.; Jamieson, R. (Southern California Edison, James Howden and Company Ltd.). (February 1988). Dynamic Response of a 330-kW Horizontal Axis Wind Turbine Generator. SERI/STR-217-3203. Golden, CO: Solar Energy Research Institute; 103 pp.

Wright, A.D.; Buhl, M.L., Jr.; Thresher, R.W. (January 1988). FLAP Code Development and Validation. SERITR-217-3125. Golden, CO: Solar Energy Research Institute.

Hansen, A.C. (University of Utah). (January 1987). The TEETER Program-Analysis and User's Guide. SERI/STR-217-3050. Golden, CO: Solar Energy Research Institute.

Lobitz, D.W.; Ashwill, T.D. (1986). Aeroelastic Effects in the Structural Dynamic Analysis of Vertical Axis Wind Turbines. SAND-85-0957. Albuquerque, NM: Sandia National Laboratories; 25 pp. (NTIS no. DE86009542/XSP). 


\section{Airfoil development and blade design}

Tangler, J.L.; Somers, D.M. (1995). NREL Airfoil Families for HAWTS. NREL/TP-442-7109. Golden, CO: National Renewable Energy Laboratory; 10 pp.

Tangler, J.L.; Smith, B.; Kelley, N.D.; Jager, D. (1992). Measured and Predicted Rotor Performance for the SERI Advanced Wind Turbine Blades. NREL/TP-257-4594. Golden, CO: National Renewable Energy Laboratory. (NTIS no. DE92001215).

Tangler, J.L.; Smith, B.; Jager, D. (1992). SERI Advanced Wind Turbine Blades. NREL/TP-257-4492. Golden, CO: National Renewable Energy Laboratory. (NTIS no. DE92001216).

Clark, R.N.; Davis, R.G. (1991). "Performance Changes Caused by Rotor Blade Surface Debris." Windpower '91 Conference; September 24-27, 1991; Palm Springs, California. Washington, DC: American Wind Energy Association; pp. 470-474.

Eppler, R. (1990). Airfoil Design and Data. New York, NY: Springer-Verlag; 562 pp.

Stoddard, F.S. (1990). "Wind Turbine Blade Technology: A Decade of Lessons Learned." Energy and the Environment, Into the 1990's, 1st World Renewable Energy Congress. Edited by A.A.M. Sayigh. Vol. 3. Tarrytown, NY: Pergamon Press; 1570 pp.

Tangler, J.L. (1987). Status of the Special-Purpose Airfoil Families. SERI/TP-217-3264. Golden, CO: Solar Energy Research Institute; 9 pp. (NTIS no. DE88001124/XSP).

Kocurek, D. (Computational Methodology Associates). (June 1987). Lifting Surface Performance Analysis for Horizontal Axis Wind Turbines. SERI/STR-217-3163. Golden, CO: Solar Energy Research Institute; 319 pp. (NTIS no. DE87001176).

Yekutieli, O.; Clark, R.N. (1987). "Influence of Blade Surface Roughness on the Performance of Wind Turbines." 6th ASME Wind Energy Symposium. New York, NY: American Society of Mechanical Engineers; pp. 181-188.

Ashwill, T.D.; Leonard, T.M. (September 1986). Developments in Blade Shape Design for a Darrieus Vertical Axis Wind Turbine. SAND-86-1263. Albuquerque, NM: Sandia National Laboratories; 21 pp.

Ostowari, C.; Niak, D. (Texas A\&M University). (January 1985). Post-Stall Wind Tunnel Datafor NACA 44xx Series Airfoil Sections. SERI/STR-217-2559. Golden, CO: Solar Energy Research Institute.

Klimas, P.C. (November 1984). Tailored Airfoils for Vertical Axis Wind Turbines. SAND-84-1062. Albuquerque, NM: Sandia National Laboratories; 12 pp.

Miley, S.J. (Texas A\&M University). (December 1984). Addendum to a Catalog of Low Reynolds Number Airfoil Data for Wind Turbine Applications. SERI/STR-217-2584. Golden, CO: Solar Energy Research Institute.

Miley, S.J. (Texas A\&M University). (February 1982). A Catalog of Low Reynolds Number Airfoil Data for Wind Turbine Applications. RFP-3387. Golden, CO: Rocky Flats Wind Energy Research Center; 615 pp. (NTIS no. DE82021712). 


\section{Drivetrain, generators, and controls}

Miller, L.S. (Wichita State University). (1995). Experimental Investigation of Aerodynamic Devices for Wind Turbine Rotational Speed Control. NREL/TP-441-6913. Golden, CO: National Renewable Energy Laboratory; $48 \mathrm{pp}$.

Lauw, H.K.; Weigand, C.H.; Marckx, D.A. (Electronic Power Conditioning, Inc.). (October 1993). Variable-Speed Wind System Design: Final Report. DOE/BP/99893-TI. Washington, DC: U.S. Department of Energy; 81 pp. (NTIS no. DE94006210).

Fardoun, A.A.; Fuchs, E.F. (University of Colorado); Carlin, P.W. (1993). "A Variable-Speed, DirectDrive Transmission Wind Power Plant." Windpower '93 Conference; July 12-16, 1993; San Francisco, California. Washington, DC: American Wind Energy Association; pp. 134-141.

Torrey, D.A. (Rensselaer Polytechnic Institute); Childs, S.E. (Atlantic Orient Corporation). (1993). "Development of Variable-Reluctance Wind Generators." Windpower '93 Conference; July 12-16, 1993; San Francisco, California. Washington, DC: American Wind Energy Association; pp. 258-265.

Schluter, L.L. (Sandia National Laboratories); Vachon, W.A. (W.A. Vachon \& Associates). (1993) "Optimizing Wind Turbine Control System Parameters." Windpower '93 Conference; July 12-16, 1993; San Francisco, California. Washington, DC: American Wind Energy Association; pp. 175-182.

Childs, S.E.; Hughes, P.S.; Saeed, A. (Atlantic Orient Corporation). (1993). "Development of a Dynamic Brake Model." Windpower '93 Conference; July 12-16, 1993; San Francisco, California. Washington, DC: American Wind Energy Association; pp. 366-373.

Vachon, W.A. (January 1990). "Smart Control Algorithms for Operation of Variable-Speed Wind Turbines." Proceedings of the Ninth ASME Wind Energy Symposium. Edited by D.E. Berg. SED-Vol. 9. New York, NY: American Society of Mechanical Engineers.

McNiff, B.P. (Second Wind Inc.); Musial, W.P. (National Renewable Energy Laboratory); Errichello, R. (GEARTECH). (September 1990). "Gear Fatigue Life Variations for Different Wind Turbine Braking Strategies." Windpower '90 Conference; September 24-28, 1990; Washington, DC. Washington, DC: American Wind Energy Association; 10 pp. (NTIS no. DE91002161).

Herrera, J.I.; Reddoch, T.W. (Electrotek Concepts, Inc.). (February 1988). Analysis of the Electrical Harmonic Characteristics of a Slip Recovery Variable Speed Generating System for Wind Turbine Applications. SERI/STR-217-3268. Golden, CO: Solar Energy Research Institute.

Herrera, J.I.; Reddoch, T.W. (Electrotek Concepts, Inc.). (February 1988). Analysis of the Electrical Harmonic Characteristics of a Westinghouse Variable Speed Generating System for Wind Turbine Applications. SERI/STR-217-3133. Golden, CO: Solar Energy Research Institute.

Reactive Power Management Device Assessment. (University of Washington). (August 1987). EPRI-AP-5210. Palo Alto, CA: Electric Power Research Institute; 96 pp.

Specification of Test Equipment for Power Electronic Drive Research. (OMNION Power Engineering Corporation). (1987). AP-5219. Palo Alto, CA: Electric Power Research Institute; 112 pp. 
Electrical Behavior of Wind Power Stations. (Zaininger Engineering Company, Inc.). (1992). Windpower '92 Conference; October I9-23, I992; Seattle, Washington. Washington DC: American Wind Energy Association.

\section{Fatigue and failure analysis}

Veers, P.S. (Sandia National Laboratories); Lange, C.H.; Winterstein, S.R. (Stanford University). (1993). "FAROW: A Tool for Fatigue and Reliability of Wind Turbines." Windpower '93 Conference; July I2-I6, I993; San Francisco, California. Washington, DC: American Wind Energy Association; pp. 342-349.

Kelley, N.D.; Desrochers, G.; Tangler, J.L.; Smith, B. (October 1992). A Discussion of the Results of the Rainflow Counting of a Wide Range of Dynamics Associated with the Simultaneous Operation of Adjacent Wind Turbines. NREL/TP-442-5159. Golden, CO: National Renewable Energy Laboratory.

Mandell, J.F.; Reed, R.M.; Samborsky, D.D. (Montana State University). (August 1992). Fatigue of Fiberglass Wind Turbine Blade Materials. SAND-92-7005. Albuquerque, NM: Sandia National Laboratories; 61 pp. (NTIS no. DE93005381).

Masse, B.; Pastorel, H. (1992). Stress Calculation for the Sandia 34-Meter Wind Turbine Using the Local Circulation Method and Turbulent Wind. SAND-91-7012. Albuquerque, NM: Sandia National Laboratories.

Ashwill, T.D.; Sutherland, H.J.; Veers, P.S. (January 1990). "Fatigue Analysis of the Sandia 34-meter Vertical Axis Wind Turbine." Proceedings of the Ninth ASME Wind Energy Symposium. Edited by D.E. Berg. SED-Vol. 9. New York, NY: American Society of Mechanical Engineers.

International Recommended Practices for Wind Energy Conversion Systems Testing: 3. Fatigue Characteristics; I. Edition I984. (1984). Edited by J.M.W. Dekker, E. Jensen, H.H. Ottens, S.E. Thor, and U. Hassan; $19 \mathrm{pp}$. Submitted to the Executive Committee of the International Energy Agency Program for Research and Development on Wind Energy Conversion Systems.

Waldon, C.A.; Hansen, A.C. (June 1983). Fatigue Modeling for Small Wind Systems: Basic Theory. RFP-3564. Golden, CO: Rocky Flats Wind Energy Research Center; 56 pp. (NTIS no. DE84013191).

\section{Testing and instrumentation}

Simms, D.A. (National Renewable Energy Laboratory); Cousineau, K.L. (Zond Systems, Inc.). (June 1993). A Rugged, Low-Cost, Advanced Data Acquisition System for Field Test Projects. NREL/TP-442-5617. Golden, CO: National Renewable Energy Laboratory; 11 pp. (NTIS no. DE93010041).

Connell, J.R. (Colorado State University); Morris, V.R. (1991). Calibration Data for Improved Correction of UVW Propeller Anemometers. PNL-7824. Richland, WA: Pacific Northwest Laboratory.

Simms, D.A.; Butterfield, C.P. (April 1990). A Low-Cost PC-Based Telemetry Data-Reduction System. SERI/TP-257-3737. Golden, CO: Solar Energy Research Institute. 


\section{Recommended practices for testing and analysis}

Safety of Wind Turbine Generator Systems. (1994). Draft International Standard: TC-88. Geneva, Switzerland: International Electrotechnical Commission.

Wind Turbines: Performance Test Codes. (1989). ASME/ANSI PRC 42-1988. New York, NY: American Society of Mechanical Engineers; $61 \mathrm{pp}$.

Standard Performance Testing of Wind Energy Conversion Systems. (1988). AWEA Standard: AWEA 1.1-1988. Arlington, VA: American Wind Energy Association; 32 pp.

Recommended Practices for Wind Turbine Testing: 4. Acoustics. Measurement of Noise Emission from Wind Energy Conversion Systems (WECS); 2. Edition 1988. (1988). Edited by S. Ljunggren, and A. Gustafsson; 23 pp. Submitted to the Executive Committee of the International Energy Agency Program for Research and Development on Wind Energy Conversion Systems.

Recommended Practices for Wind Turbine Testing and Evaluation: 8. Glossary of Terms; Issue 1, March 1987. (March 1987). Edited by G. Elliot; 11 pp. Submitted to the Executive Committee of the International Energy Agency Program for Research and Development on Wind Energy Conversion Systems.

International Recommended Practices for Wind Energy Conversion Systems Testing: 3. Fatigue Characteristics; 1. Edition 1984. (1984). Edited by J.M.W. Dekker, E. Jensen, H.H. Ottens, S.E. Thor, and U. Hassan; $19 \mathrm{pp}$. Submitted to the Executive Committee of the International Energy Agency Program for Research and Development on Wind Energy Conversion Systems.

Recommended Practices for Wind Turbine Testing: 7. Quality of Power. Single Grid-Connected WECS; 1. Edition 1984. (1984). Edited by L.J. Ballard, and R.H. Sivansborough; 24 pp. Submitted to the Executive Committee of the International Energy Agency Program for Research and Development on Wind Energy Conversion Systems.

Recommended Practices for Wind Turbine Testing: 2. Estimation of Cost of Energy from Wind Energy Conversion Systems; 1. Edition 1983. (1983). Edited by J. Nitteberg, A.A. deBoer, and P.B. Simpson; 23 pp. Submitted to the Executive Committee of the International Energy Agency Program for Research and Development on Wind Energy Conversion Systems. (NTIS no. DE84901132).

Recommended Practices for Wind Turbine Testing: 1. Power Performance Testing; 1. Edition 1982. (1982). Edited by S. Frandsen, A.R. Trenka, and B. Pedersen; 17 pp. Submitted to the Executive Committee of the International Energy Agency Program for Research and Development on Wind Energy Conversion Systems. (NTIS no. DE84901417). 


\section{Chapter 5 Applications}

\section{General}

Wind Energy in the U.S.: A State By State Survey. (current). Washington, DC: American Wind Energy Association; $183 \mathrm{pp}$.

Spera, D.A. (May 1994). Wind Turbine Technology: Fundamental Concepts of Wind Turbine Engineering. 100368. Fairfield, NJ: American Society of Mechanical Engineers; 700 pp.

American Wind Energy Association. (1994). American Wind Energy Association's 1994 Membership Directory. Washington, DC: American Wind Energy Association; 42 pp.

Interstate Renewable Energy Council; Solar Energy Industries Association; Sandia National Laboratories. (1993). Procurement Guide for Renewable Energy Systems; 140 pp. Available from American Solar Energy Society, 2400 Central Avenue, G-1, Boulder, CO 80301.

Gipe, P. (1993). Wind Power for Home \& Business. Post Mills, VT: Chelsea Green Publishing Company; 413 pp.

Recommended Practice for the Installation of Wind Energy Conversion Systems. (1989). AWEA Standard: AWEA 6.1-1989. Washington, DC: American Wind Energy Association; 48 pp.

Jaras, T. (1988). Wind Energy 1988: Wind Turbine Shipments and Applications. Great Falls, VA: Stadia, Inc. (Wind Data Center); 316 pp.

Jaras, T. (1987). Wind Energy 1987: Wind Turbine Shipments and Applications. Great Falls, VA: Stadia, Inc. (Wind Data Center).

Status of Commercial Wind Power: 1986 Survey. (Strategies Unlimited). (1986). AP-5084. Palo Alto, CA: Electric Power Research Institute; 308 pp.

Moment, R.L. (February 1983). Performance and Size Estimating for Wind Systems. RFP-3586. Golden, CO: Rockwell International Corporation; 69 pp.

Park, J. (1981). The Wind Power Book. Palo Alto, CA: Chesire Books; 253 pp.

Wind Energy Mission Analysis. (February 1977). COO/2578-1/1-3. Philadelphia, PA: General Electric Company.

Wind Energy Mission Analysis. (April 1976). SAN-1075-1/1-3. Burbank, CA: Lockheed-California Company. 


\section{Operation, maintenance, and performance of large wind power plants}

Experiences with Commercial Wind Energy Development in Hawaii. (R. Lynette \& Associates, Inc.). (1993). EPRI-TR-102169. Palo Alto, CA: Electric Power Research Institute; 116 pp.

Clark, R.N.; Davis, R.G. (1993) "Performance of an Enertech 44 During 11 Years of Operation." Windpower '93 Conference; July 12-16, 1993; San Francisco, California. Washington, DC: American Wind Energy Association; pp. 204-212.

California Energy Commission. (1992). 1992 Energy Technology Status Report. P500-92-007E. Sacramento, CA: California Energy Commission; 82 pp.

Assessment of Wind Power Station Performance and Reliability. (R. Lynette \& Associates, Inc.). (1992). EPRI-TR-100705. Palo Alto, CA: Electric Power Research Institute; 144 pp.

Experiences With Commercial Wind Turbine Design, Volumes 1 and 2. (R. Lynette \& Associates, Inc.). (1989). RP-1590-12. Palo Alto, CA: Electric Power Research Institute; Vol. 1; 204 pp.; Vol. 2; 100 pp.

Solano MOD-2 Wind Turbine Operating Experience Through 1988. (Pacific Gas and Electric Company). (1989). RP-1590-06. Palo Alto, CA: Electric Power Research Institute; 60 pp.

Standard Performance Testing of Wind Energy Conversion Systems. (1988). AWEA Standard: AWEA 1.1-1988. Arlington, VA: American Wind Energy Association; 32 pp.

Altamont Wind Power Plant Evaluation for 1986. (Pacific Gas and Electric Company). (1988). AP-5824. Palo Alto, CA: Electric Power Research Institute; 96 pp.

\section{Integrating wind systems with utility electric grids}

Wind as a Utility-Grade Supply Resource: A Planning Framework for the Pacific Northwest. (Decision Focus, Inc.; Litchfield Consulting Group). (1993). EPRI-TR-102094. Palo Alto, CA: Electric Power Research Institute; 44 pp.

Small System Performance Under High Wind Plant Penetration. (Electrotek Concepts, Inc.). (1993). EPRI-TR-102784. Palo Alto, CA: Electric Power Research Institute; 106 pp.

Integrating an Ever-Changing Resource. (1992). Produced under the auspices of the Utility Wind Interest Group; published by the Electric Power Research Institute; 4 pp. Available from the National Renewable Energy Laboratory, Golden, CO 80401.

Wade, J.E.; Walker, S.N.; Baker, R.W. (Oregon State University). (February 1990). Integration of Wind Energy into the Electrical Utility System: An Overview of the Issues. DOE/BP/63406-11. Washington, DC: U.S. Department of Energy; 35 pp. (NTIS no. DE90009468/XAB).

Younkins, T.D. (April 1987). Methodology for Control and Operation of Wind Arrays in Utility Systems: Final Report. ORNL/Sub/81-7242/1. Oak Ridge, TN: Oak Ridge National Laboratory; 290 pp. (NTIS no. DE87008546). 
Recommended Practices for Wind Turbine Testing: 7. Quality of Power. Single Grid-Connected WECS; 1. Edition 1984. (1984). Edited by L.J. Ballard, and R.H. Sivansborough; 24 pp. Submitted to the Executive Committee of the International Energy Agency Program for Research and Development on Wind Energy Conversion Systems.

McCabe, T.; Henry, G.; Tennis, M.W.; Goldenblatt, M. (January 1984). Early Utility Experience with Wind Power Generation. EPRI-AP-3233. Vol. 1; 50 pp.; Vol. 2; 118 pp.; Vol. 3; 150 pp. Palo Alto, CA: Electric Power Research Institute.

Chan, S.M.; Curtice, D.; Chang, S.K. (October 1983). Methods for Wind Turbine Dynamic Analysis. EPRI-AP-3259. Palo Alto, CA: Electric Power Research Institute; 272 pp.

Goldenblatt, M.K.; Wegley, H.L. (March 1983). Analysis of the Effects of Integrating Wind Turbines into a Conventional Utility: A Case Study. PNL-3962-Rev. Richland, WA: Pacific Northwest Laboratory; $66 \mathrm{pp}$.

Kaupent, B.M. (February 1983). Assessment of Distributed Wind Power Systems. EPRI-AP-2882. Palo Alto, CA: Electric Power Research Institute; 364 pp.

Marah, F.; Dlott, E.H.; Korn, D.H. (1982). Wind Power for the Electric-Utility Industry. Lexington, MA: DC Heath and Company, Lexington Books; 159 pp.

Wind-Hydroelectric Energy Project-Wyoming. (August 1982). Washington, DC: U.S. Department of the Interior, Bureau of Reclamation; $181 \mathrm{pp}$.

Reddock, T.W.; Barnes, P.R.; Lawler, J.S.; Skroski, J.C. (May 1982). "Strategies for Minimizing Operational Impacts of Large Wind Turbine Arrays on Automatic Generation Control Systems." Journal of Solar Energy Engineering (104:2); New York, NY: American Society of Mechanical Engineers; pp. 65-69.

Hinrickson, E.N.; Nolan, P.J. (November 1981). Dynamics of Single- and Multi-Unit Wind Energy Conversion Plants Supplying Electric Utility Systems. DOE/ET/20466-78/1. Washington, DC: U.S. Department of Energy; 139 pp.

Wind Power Generation Dynamic Impacts on Electric Utility Systems. (November 1980). EPRI-AP-1614. Palo Alto, CA: Electric Power Research Institute; 90 pp.

Requirements Assessment of Wind Power Plants in Electric Utility Systems. (January 1979). EPRI-ER-978-SY. Palo Alto, CA: Electric Power Research Institute; 3 vols.

\section{Agricultural applications}

Nelson, V.; Gilmore, E.H.; Starcher, K. (1993). Introduction to Wind Energy. 93-3. Canyon, TX: Alternative Energy Institute; 28 pp.

Wyatt, A. (Research Triangle Institute). (1992). Wind Electric Pumping Systems: Sizing and Cost Estimation. Washington, DC: American Wind Energy Association; 39 pp. 
Clark, R.N.; Mulh, K.E. (1992). "Water Pumping for Livestock." Windpower '92 Conference; October 19-23, 1992; Seattle, Washington. Washington, DC: American Wind Energy Association; pp. 284-290.

Gilley, J.R. (July 1985). "Potential Use of Wind Power for Pumping Irrigation Water." Energy Agriculture (4:2); pp. 133-146.

Wschenbach, W. (1984). Wind Systems for Pumping Water: A Training Manual. Washington, DC: Peace Corps; 83 pp.

Stafford, R.W.; Soderholm, L.H. (March 1983). Analysis of Problems of Interfacing Wind Systems to Rural Power-Distribution Systems: Final Report. DOE/ARS-3408-20691/83/1. Ames, IA: Agricultural Research Service; 216 pp. (NTIS no. DE83013803).

Marjan, P.L.; Clark, R.N. (November 1982). Evaluation of Pumps for Wind-Driven Irrigation. DOE/ARS-7315-20741/83/1. Washington, DC: U.S. Department of Energy; 174 pp. (NTIS no. DE83013438).

Stafford, R.W.; Greeb, F.J.; Smith, M.F.; Des Chenes, C.; Weaver, N.L. (January 1981). Economic Analysis of Wind-Powered Farmhouse and Farm Building Heating Systems: Final Report. DOE/SEA-3408-20691/81/1. Washington, DC: U.S. Department of Energy; 360 pp. Available from NTIS.

Clark, R.N.; Schneider, A.D.; Nelson, V.; Gilmore, E; Barieau, R.E. (January 1981). Wind Energy for Irrigation-Wind-Assisted Pumping from Wells: Final Report. DOE/SEA-7315-20741/81/1; 60 pp. Available from NTIS.

David, M.L.; Buzenberg, R.J.; Glynn, E.F.; Johnson, G.L.; Shultis, J.K.; Wagner, J.P. (August 1979). Wind Energy Applications in Agriculture, Final Report: Executive Summary. DOE/SEA-1109-20401/79/2. Beltsville, MD: U.S. Department of Agriculture, Science and Education Administration; 210 pp.

\section{Wind/diesel hybrid systems}

University of Massachusetts. "Wind/Diesel Bibliography." Current part of Endnote Plus data base available from Niles \& Associates, Inc., 200 Hearst Street, Berkeley, CA 94709.

Wind-Diesel Systems: A Guide to the Technology and its Implementation. (1994). Edited by R. Hunter and G. Elliot. Cambridge, England: Cambridge University Press.

Baring-Gould, E.I.; Manwell, J.F.; Jeffries, W.Q.; Stein, W.M. (University of Massachusetts). (1994). "Experimental Validation of the University of Massachusetts Wind/Diesel System Simulation Code, HYBRID1." Wind Energy 1994. SED-Vol. 15. New York, NY: American Society of Mechanical Engineers; pp. 77-86.

Cramer, G.; Kleinkauf, W.; Schott, T. (1994). "Wind/Diesel/Battery Systems—Applications, Experience, Economy of Different System Configurations and Sizes." Wind Engineering (17:5); p. 228. Brentwood, England: Multi-Science Publishing Company, LTD. 
Rogers, A; Manwell, J.F.; Stein, W.M.; McGowan, J.G. (1993). "Experimental Testing of Variable Speed Diesels for Wind/Diesel System Applications." Wind Energy_1993; January 31-February 4, 1993; Houston, Texas. SED-Vol. 14. New York, NY: American Society of Mechanical Engineers.

Qi, G. "Mathematic Programming Models for Economic Design and Assessment of Wind/Diesel Systems." Windpower '93 Conference; July 12-16, 1993; San Francisco, California. Washington, DC: American Wind Energy Association; pp. 501-507.

Manwell, J.F.; McGowan, J.G. (January 1992). "Advances in Quasi-Steady Wind Diesel System Simulation." 11th ASME Wind Energy Symposium. New York, NY: American Society of Mechanical Engineers.

King, W.R.; Johnson, B.L., III. (Science Applications International Corporation) (1991). Worldwide WindDiesel Hybrid Power System Study: Potential Applications and Technical Issues. SERI/TP-257-3757. Golden, CO: Solar Energy Research Institute; 104 pp. (NTIS no. DE91002160).

Smith, J.C. (1991). Studies on the Modeling of Wind-Diesel Systems and the Integration of Wind Energy Systems with Electric Utilities. Prepared for DOE/EC Wind Energy Assessment and Review, Albuquerque, New Mexico, June 4, 1991, by Electrotek Concepts, Inc.

WindDiesel Systems Architecture Guidebook. (1991). AWEA Standard: AWEA 10.1-1991. Washington, DC: American Wind Energy Association; 41 pp.

Coleman, C. (Northern Power Systems). (1990). Wind Hybrid System Research and Testing: Telecommunications Systems. SERI/STR-257-3686. Golden, CO: Solar Energy Research Institute.

Hughes, P.S.; Johnson, B.; Sherwin, R.; Stern, W. (Atlantic Orient Corporation). (1990). System Stability and Penetration Study for Wind Diesel Hybrid Systems. SERI/STR-257-3982. Golden, CO: Solar Energy Research Institute.

Lundsager; Norgaard. (August 1988). The 55/30 kW Experimental WindDiesel System at Riso National Laboratory. RISO-M-2717. Roskilde, Denmark: Riso National Laboratory.

Dawson, R.E.; Cowan, R.A. (March 1987). Multisource Energy System Project. SERI/SP-217-2950. Golden, CO: Solar Energy Research Institute; 35 pp.

Clark, R.N. (1985). "Wind-diesel Hybrid System for Water Pumping." Proceedings of the Wind Energy Exposition and National Conference of teh American Wind Energy Association. Washington, DC: American Wind Energy Association; pp. 221-225.

Wilreker, V.F.; Stiller, P.H.; Scott, G.W.; Kruse, V.J.; Smith, R.F. (February 1984). Wind Turbine Generator Interaction with Conventional Diesel Generators on Block Island, Rhode Island. Vol. 1, Executive Summary. DOE/NASA-0354-1. Cleveland, OH: NASA Lewis Research Center; 45 pp. (NTIS no. DE84015874). 


\section{International applications}

\section{Industrialized nations}

Van Wijk, A.J.M.; Coelingh, J.P. (1993). Wind Power Potential in the OECD Countries. 93091. Utrecht, The Netherlands: Utrecht University; 35 pp.

OEM Development Corporation. (March 1993). European Wind Technology. EPRI-TR-101391. Palo Alto, CA: Electric Power Research Institute; 88 pp.

Commission of the European Community's Directorate and Fraunhofer-Institute for Solar Energy Systems (1990-1991). Eurowin-European Wind Turbine Data Base Annual Reports. Available from: American Wind Energy Association, Washington, D.C.

\section{Developing nations}

American Wind Energy Association. (1993). Small Wind Energy Systems Applications Guide. Washington, DC: American Wind Energy Association; 8 pp.

American Wind Energy Association. (1992). Wind Energy for Sustainable Development. Washington, DC: American Wind Energy Association; 22 pp.

Wyatt, A. (Research Triangle Institute). (1992). Wind Electric Pumping Systems: Sizing and Cost Estimation. Washington, DC: American Wind Energy Association; 39 pp.

Wind Energy Applications and Training Symposium. Final Report. (1990). Conference proceedings. Arlington, VA: American Wind Energy Association; 120 pp. (NTIS no. TI91006703).

Clark, R.N. (1989). "Stand-alone Wind Electric Water Pumping." Proceedings of the European Wind Energy Conference, EWEC '89. Amsterdam: Elsevier; pp. 650-654. 


\section{Chapter 6 Environmental Issues of Wind Power}

\section{General}

Wind Power and the Environment. (1993). Produced by the Utility Wind Interest Group; published by the Electric Power Research Institute; 4 pp. Golden, CO: National Renewable Energy Laboratory.

Hohmeyer, O. (1988). Social Costs of Energy Consumption. Berlin-Heidelberg, Federal Republic of Germany: Springer-Verlag; 126 pp.

Lawrence, K.A.; Strojan, C.L. (May 1980). Environmental Effects of Small Wind Energy Conversion Systems (SWECS). SERITP-743-628. Golden, CO: Solar Energy Research Institute; 16 pp.

Rogers, S.E.; Comaby, B.W.; Redman, C.W.; Sticksel, P.R.; Talle, D.A. (December 1977). Environmental Studies Related to the Operation of Wind Energy Conversion Systems. COO-0092-77/2. Columbus, OH: Battelle Columbus Laboratory; 108 pp.

\section{Wildlife}

Whalen, W. (Kenetech). (1994). "Siting Wind Plants and the Avian Issue." Windpower '94 Conference; May 9-13, 1994; Minneapolis, MN. Washington, DC: American Wind Energy Association.

Jacobs, M. (Massachusetts Department of Public Utilities). (1994). "Avian Mortality and Windpower in the Northeast." Windpower '94 Conference; May 9-13, 1994; Minneapolis, MN. Washington, DC: American Wind Energy Association.

Proceedings: Avian Interactions with Utility Structures. (December 1993). EPRI-TR-103268. Palo Alto, CA: Electric Power Research Institute; 428 pp.

Wind Turbine Effects on Avian Activity, Habitat Use, and Mortality in Altamont Pass and Solano County Wind Resource Areas, 1989-1991. (March 1992). Sacramento, CA: California Energy Commission; $145 \mathrm{pp}$.

\section{Acoustics}

Hubbard, H.H.; Shepherd, K.P. (June 1991). "Aeroacoustics of Large Wind Turbines." Journal of the Acoustic Society of America (89:6); pp. 2495-2508.

Procedure for Measurement of Acoustic Emissions from Wind Turbine Generator Systems. Vol. I: First Tier. (1989). AWEA Standard: AWEA 2.1-1989. Washington, DC: American Wind Energy Association; $35 \mathrm{pp}$.

Recommended Practices for Wind Turbine Testing: 4. Acoustics. Measurement of Noise Emission from Wind Energy Conversion Systems (WECS); 2. Edition 1988. (1988). Edited by S. Ljunggren, and A. Gustafsson; 23 pp. Submitted to the Executive Committee of the International Energy Agency Program for Research and Development on Wind Energy Conversion Systems. 
Kelley, N.D.; McKenna, H.E.; Jacobs, E.W.; Hemphill, R.R.; Birkenheuer, N.J. (January 1988). The MOD-2 Wind Turbine: Aeroacoustical Noise Sources, Emissions, and Potential Impact. SERI/TR-217-3036. Golden, CO: Solar Energy Research Institute.

Kelley, N.D. (November 1987). A Proposed Metric for Assessing the Potential of Community Annoyance from Wind Turbine Low-Frequency Noise Emissions. SERI/TP-217-3261. Golden, CO: Solar Energy Research Institute.

Kelley, N.D. (February 1985). Acoustic Noise Associated with the MOD-1 Wind Turbine: Its Source, Impact, and Control. SERI/TR-635-1166. Golden, CO: Solar Energy Research Institute.

Hemphill, R.R. (May 1983). An Acoustic Ranging Technique with Application Assessment of LowFrequency Acoustic Noise of Wind Turbines. SERI/TP-215-1954. Golden, CO: Solar Energy Research Institute.

Stephens, D.G.; Shepherd, K.P.; Hubbard, H.H.; Grosveld, F.W. (March 1982). Guide to the Evaluation of Human Exposure to Noise from Large Wind Turbines. NASATM-83288. Hampton, VA: NASA Langley Research Center; 70 pp.

\section{Electromagnetic interference}

Recommended Practices for Wind Turbine Testing and Evaluation; 5. Electromagnetic-interference; Preparatory Information; Issue 1, February 1986. (1986). Edited by R.J. Chignell; 16 pp. Submitted to the Executive Committee of the International Energy Agency Program for Research and Development on Wind Energy Conversion Systems.

Sengupta, D.L.; Senior, T.B.; Ferris, J.E. (University of Michigan). (1983). Large Wind Turbine Siting Handbook: Television Interference Assessment, Final Subcontract Report. SERI/STR-215-1879. Golden, CO: Solar Energy Research Institute.

Sengupta, D.L.; Senior, T.B.; Ferris, J.E. (University of Michigan). (November 1983). Television Interference Measurements Near the Mod-2 WT Array at Goodnoe Hills, Washington: A Subcontract Report. SERISTR-211-2086. Golden, CO: Solar Energy Research Institute; 64 pp.

Sengupta, D.L.; Senior, T.B.; Ferris, J.E. (January 1983). Measurements of Television Interference Caused by a Vertical Axis Wind Machine. SERI/STR-215-1881. Golden, CO: Solar Energy Research Institute. $90 \mathrm{pp}$.

Sengupta, D.L.; Senior, T.B.; Ferris, J.E. (January 1983). Study of Television Interference by Small Wind Turbines: Final Subcontract Report. SERI/STR-215-1881. Golden, CO: Solar Energy Research Institute; $47 \mathrm{pp}$.

Senior, T.B.; Sengupta, D.L.; Ferris, J.E. (February 1977). TV and FM Interference by Windmills. EOE/TIC-11348. Washington, DC: Energy Research and Development Administration. 


\section{Chapter 7 Institutional Issues}

\section{Legal and regulatory issues}

Wind Energy in the U.S.: A State By State Survey. (current). Washington, DC: American Wind Energy Association; 183 pp.

Wind Energy Regulators Handbook. (1994). Washington, DC: American Wind Energy Association.

Ing, E.T.C. (July 1993). "Making Sense of the Federal Tax Code: Incentives for Windfarm Development." Windpower '93 Conference; July 12-16, 1993; San Francisco, California. Washington, DC: American Wind Energy Association; pp. 40-46.

Hamrin, J.; Rader, N. (1993). Investing in the Future: A Regulator's Guide to Renewables. Washington, DC: National Association of Regulatory Utility Commissioners; 322 pp.

Removing Barriers to Wind Energy: Directions for State Regulatory Action. (1993). Washington, DC: American Wind Energy Association; 16 pp.

Oberg, K.W. (ESI Energy, Inc.). (1992). "Impact of Production Incentives on Financing Wind Energy Projects." Windpower '92 Conference; October 19-23, 1992; Seattle, Washington. Washington, DC: American Wind Energy Association; pp. 167-172.

Bain, D. (Oregon State Department of Energy); Bloomquist, R.G. (Washington State Energy Office). (December 1992). Wind/Solar: A Regulatory Guide to Leasing, Permitting, and Licensing in Idaho, Montana, Oregon, and Washington. DOE/BP-2015. Bonneville Power Administration. Washington, DC: U.S. Department of Energy; 177 pp. (NTIS no. DE93012372).

PURPA Handbook. (1992). Washington, DC: American Wind Energy Association; 14 pp.

Renewable Energy and Utility Regulation. (April 1991). National Association of Regulatory Utility Commissioners. DOE/SE/28301-T6. Washington, DC: U.S. Depanment of Energy; 48 pp. (NTIS no. DE92013626).

Lornell, R.; Schaller, D.A. (January 1982). Small Power Production and Wind Energy: Regulatory Actions Under PURPA. SERI/SP-635-794. Golden, CO: Solar Energy Research Institute; 64 pp.

Dodge, D.M.; Lawless-Butterfield, C. (May 1982). Small Wind Systems Zoning Issues and Approaches. RFP-3386. Golden, CO: Rocky Flats Wind Energy Research Center; 60 pp. (NTIS no. DE83007195).

Phillips, P.D. (May/June 1979). "NEPA and Alternative Energy: Wind as a Case Study." Solar Law Reporter (1:1); pp. 29-54. Golden, CO: Solar Energy Research Institute. 


\section{Safety issues}

Safety of Wind Turbine Generator Systems. (1994). Draft International Standard: TC-88. Geneva, Switzerland: International Electrotechnical Commission.

Recommended Practice for the Siting of Wind Energy Conversion Systems. (1993). AWEA Standard: AWEA 8.2-1993. Washington, DC: American Wind Energy Association; 24 pp.

National Electrical Code Handbook. (1993). NFPA No. 70HB93. Quincy, MA: National Fire Protection Association. (ISBN 0-87765-384-4).

Occupational Safety and Health Standards for General Industry. (1989). Chicago, IL: Commerce Clearing House, Inc.

Recommended Practice for the Installation of Wind Energy Conversion Systems. (1989). AWEA Standard: AWEA 6.1-1989. Washington, DC: American Wind Energy Association.

Kurtz, E.B.; Shoemaker, T.M. (1986). The Lineman's and Cableman's Handbook, Seventh Edition. New York, NY: McGraw-Hill Book Company. (ISBN 0-07 035686-6).

Bankaitis, H. (1981). Lightning Accommodation Systems for Wind Turbine Generator Safety. NASA TM-82601. Cleveland, OH: NASA Lewis Research Center; 13 pp.

\section{Standards and recommended practices}

Issued by the American Wind Energy Association:

Recommended Practice for the Siting of Wind Energy Conversion Systems. (1993). AWEA Standard: AWEA 8.2-1993. Washington, DC: American Wind Energy Association; pp. 24.

WindDiesel Systems Architecture Guidebook. (1991). AWEA Standard: AWEA 10.1-1991. Washington, DC: American Wind Energy Association.

Procedure for Measurement of Acoustic Emissions from Wind Turbine Generator Systems. Vol. I: First Tier. (1989). AWEA Standard: AWEA 2.1-1989. Washington, DC: American Wind Energy Association.

Recommended Practice for the Installation of Wind Energy Conversion Systems. (1989). AWEA Standard: AWEA 6.1-1989. Washington, DC: American Wind Energy Association.

Standard Performance Testing of Wind Energy Conversion Systems. (1988). AWEA Standard: AWEA 1.1-1988. Arlington, VA: American Wind Energy Association; 32 pp.

Design Criteria Recommended Practices: Wind Energy Conversion Systems. (1988). AWEA Standard: AWEA 3.1-1988. Arlington, VA: American Wind Energy Association; 25 pp.

Standard Procedures for Meteorological Measurements at a Potential Wind Turbine Site. (1986). AWEA Standard: AWEA 8.1-1986. Alexandria, VA: American Wind Energy Association; 18 pp. 
Wind Energy Conversion Systems Terminology. (1985). AWEA Standard 5.1-1985. Alexandria, VA: American Wind Energy Association; 11 pp.

Issued by the International Energy Agency Program for Research and Development on Wind Energy Conversion Systems:

Recommended Practices for Wind Turbine Testing: 4. Acoustics. Measurement of Noise Emission from Wind Energy Conversion Systems (WECS); 2. Edition 1988. (1988). Edited by S. Ljunggren, and A. Gustafsson; 23 pp. Submitted to the Executive Committee of the International Energy Agency Program for Research and Development on Wind Energy Conversion Systems.

Recommended Practices for Wind Turbine Testing and Evaluation: 8. Glossary of Terms; Issue 1, March 1987. (March 1987). Edited by G. Elliot; 11 pp. Submitted to the Executive Committee of the International Energy Agency Program for Research and Development on Wind Energy Conversion Systems.

Recommended Practices for Wind Turbine Testing and Evaluation: 5. Electromagnetic-interference; Preparatory Information; Issue 1, February 1986. (1986). Edited by R.J. Chignell; 16 pp. Submitted to the Executive Committee of the International Energy Agency Program for Research and Development on Wind Energy Conversion Systems.

International Recommended Practices for Wind Energy Conversion Systems Testing: 3. Fatigue Characteristics; 1. Edition 1984. (1984). Edited by J.M.W. Dekker, E. Jensen, H.H. Ottens, S.E. Thor, and U. Hassan; 19 pp. Submitted to the Executive Committee of the International Energy Agency Program for Research and Development on Wind Energy Conversion Systems.

Recommended Practices for Wind Turbine Testing: 7. Quality of Power. Single Grid-Connected WECS; 1. Edition 1984. (1984). Edited by L.J. Ballard, and R.H. Sivansborough; 24 pp. Submitted to the Executive Committee of the International Energy Agency Program for Research and Development on Wind Energy Conversion Systems.

Recommended Practices for Wind Turbine Testing: 2. Estimation of Cost of Energy from Wind Energy Conversion Systems; 1. Edition 1983. (1983). Edited by J. Nitteberg, A.A. deBoer, and P.B. Simpson; 23 pp. Submitted to the Executive Committee of the International Energy Agency Program for Research and Development on Wind Energy Conversion Systems. (NTIS no. DE84901132).

Recommended Practices for Wind Turbine Testing: 1. Power Performance Testing; 1. Edition 1982. (1982). Edited by S. Frandsen, A.R. Trenka, and B. Pedersen; 17 pp. Submitted to the Executive Committee of the International Energy Agency Program for Research and Development on Wind Energy Conversion Systems. (NTIS no. DE84901417).

Issued by the American Society of Mechanical Engineers (ASME) and the American National Standards Institute (ANSI):

Wind Turbines: Performance Test Codes. (1989). ASME/ANSI PRC 42-1988. New York, NY: The American Society of Mechanical Engineers; $61 \mathrm{pp}$. 
Issued by the International Electrotechnical Commission:

Safety of Wind Turbine Generator Systems. (1994). Draft International Standard: TC-88. Geneva, Switzerland: International Electrotechnical Commission. 


\section{Chapter 8 Wind Energy Systems Development}

\section{Turbine Development Program}

Lynette, R. (R. Lynette \& Associates, Inc.). (1995). Advanced Wind Turbine Conceptual Study Final Report. NREL/TP-441-6924. Golden, CO: National Renewable Energy Laboratory; 336 pp. plus appendices.

Atlantic Orient Corporation. (1994). Advanced Wind Turbine Design Studies, Advanced Conceptual Study, Final Report. NREL/TP-442-4740. Golden, CO: National Renewable Energy Laboratory; 245 pp.

Bell, B. (FloWind Corporation). (1994). "FloWind's Advanced EHD-Series Wind Turbine." CanWEA Conference; October 17-19, 1994; Regina, Saskatchewan, Canada. Ottawa, Ontario: Canadian Wind Energy Association.

Hughes, P.S. (Atlantic Orient Corporation). (1994). "Component Testing for the AOC 15-50." Windpower '94 Conference; May 10-13, 1994; Minneapolis, Minnesota. Washington, DC: American Wind Energy Association.

Laxson, A.S.; Dodge, D.M.; Flowers, L.T.; Loose, R.; Goldman, P. (1993). An Overview of DOE's Wind Turbine Development Programs. NREL/TP-441-5761. Golden, CO: National Renewable Energy Laboratory; 8 pp.

Coleman, C. (Northern Power Systems). (1993). "Northern Power Systems Advanced Wind Turbine Development Program." Windpower '93 Conference; July 12-16, 1993; San Francisco, California. Washington, DC: American Wind Energy Association; pp. 152-154.

Swift, A. (University of Texas); Hock, S.M.; Thresher, R.W. (National Renewable Energy Laboratory). (February 1993). "Advanced Wind Turbine Performance and Cost Projections: A Configuration Survey." ASME 16th Annual Energy-Sources Technology Conference; Houston, Texas. Fairfield, NJ: American Society of Mechanical Engineers.

Rodgers, J. (R. Lynette \& Associates, Inc.). (1992). "WC-86 Advanced Wind Turbine Development." 11th ASME Wind Energy Symposium, January 1992. Fairfield, NJ: American Society of Mechanical Engineers.

Advanced Wind Turbines: Electricity for the 1990s and Beyond. (April 1992). 8 pp. Available from the National Renewable Energy Laboratory, Golden, CO.

\section{Development of small systems}

Lynch, J.; Coleman, C.; Mayer, D.J. (North Wind Power Company, Inc.). (January 1983). North Wind 4-kW Wind System Development, Phase II-Fabrication and Test. RFP-3516. Golden, CO: Solar Energy Research Institute. (NTIS no. DE83017774) 
Lynch, J.; Coleman, C.; Mayer, D.J. (North Wind Power Company, Inc.). (May 1982). North Wind 4-kW Wind System Development, Phase I-Design and Analysis. RFP-3424. Golden, CO: Solar Energy Research Institute. (NTIS no. DE83012271).

Zickefoose, C.R. (Enertech Corporation). (December 1982). Enertech 15-kW Wind System Development; Phase II-Fabrication and Test. RFP-3515. Golden, CO: Rocky Flats Wind Energy Research Center; 58 pp. (NTIS no. DE83017773).

Enertech Corporation. (September 1981). Enertech 15-kW Wind System Development; Phase I-Design and Analysis, Volume II. Technical Report. RFP-3341. Golden, CO: Solar Energy Research Institute. (NTIS no. DE82018403).

Mayer, D.J.; Norton, J.H., Jr. (North Wind Power Company, Inc.). (June 1982). North Wind 2-kW HighReliability Wind System, Phase II-Fabrication and Test. RFP-3426. Golden, CO: Wind Systems Program, Rockwell International Corporation; 43 pp. (NTIS no. DE83007196).

Mayer, D.J.; Norton, J.H., Jr. (North Wind Power Company, Inc.). (July 1981). North Wind Power Company 2-Kilowatt High Reliability Wind System, Phase I-Design and Analysis Technical Report. RFP-3310. Golden, CO: Solar Energy Research Institute. (NTIS no. DE82015124).

Proceedings: Small Wind Turbine Systems. (1981). May 12-14, 1981; Boulder, CO. SERI/CP-635-1212. Golden, CO: Solar Energy Research Institute; 355 pp.

Adler, F.M. et al. (Grumman Energy Systems, Inc.). (November 1980). Grumman WS33 Wind System, Prototype Construction and Testing Phase II Technical Report. RFP-3288. Golden, CO: Solar Energy Research Institute. (NTIS no. DE82017756).

Adler, F.M. et al. (Grumman Energy Systems, Inc.). (March 1980). Development of an 8-kW Wind Turbine Generator for Residential Type Applications, Phase I-Design and Analysis Technical Report. RFP-3007. Golden, CO: Solar Energy Research Institute. (NTIS no. RFP-3007[V.2]).

Cheney, M.C. et al. (United Technologies Research Center). (June 1979). Development of an 8-kW Wind Turbine Generator for Residential Type Applications, Phase I-Design and Analysis. RFP-3006. Golden, CO: Solar Energy Research Institute. (NTIS no. DOE/DP/03533-T1[V.2]).

Cheney, M.C. (United Technologies Research Center). (February 1982). UTRC 15-kW Wind System Development, Phase II-Fabrication and Testing. RFP-3358. Golden, CO: Solar Energy Research Institute. (NTIS no. DE83012274).

Taylor, R.B.; Cheney, M.C. (United Technologies Research Center). (December 1981). UTRC 15-kW Wind System Development, Phase I-Design and Analysis. RFP-3331. Golden, CO: Solar Energy Research Institute. (NTIS no. DE82021710).

\section{Development of large horizontal-axis systems}

MOD-5B Wind Turbine System Final Report. Vol. I: Executive Summary. DOE/NASA 0200-3. Vol. II: Detailed Report. DOE/NASA 0200-4. Vol. III: Acceptance Testing. (March 1988). Cleveland: NASA Lewis Research Center. 
MOD-5A Wind Turbine Generator Program Design Report. Vol. 1: Executive Summary. DOE/NASA/0153-1; 63 pp. Vol. II: Conceptual and Preliminary Design, Books 1 and 2. DOE/NASA/0153-2; 661 pp. Vol. III: Final Design and System Description, Books 1 and 2. DOE/NASA/0153-3; 1145 pp. Vol. IV: Drawings and Specification, Books 1-5. DOE/NASA/0153-4; 2754 pp. (August 1984). Cleveland, OH: NASA Lewis Research Center.

Ancona, D.F.; Spera, D.A.; MOD-2 Final Technical Report. (May 1994). NREL/SP-440-5177. Golden, CO: National Renewable Energy Laboratory; 124 pp. (NTIS no. DE93000014).

MOD-2 Wind Turbine System Development; Final Report. Volume 1. Executive Summary. DOE/NASA-0002-821/1; 35 pp. (NTIS no. DE83015190). Volume II. Detailed Report. DOE/NASA-0002-821/2; 191 pp. (NTIS no. DE83017707). (Boeing Engineering and Construction). (September 1982). Cleveland, OH: NASA Lewis Research Center.

Collins, J.L.; Shaltens, R.K.; Poor, R.H.; Barton, R.S. (April 1982). Experience and Assessment of the DOE-NASA MOD-1 2000-Kilowatt Wind Turbine Generator at Boone, North Carolina. DOE/NASA/20366-2. (NTIS no. DE82020317). Cleveland, OH: NASA Lewis Research Center; 53 pp.

Wilreker, V.F.; Stiller, P.H.; Scott, G.W.; Kruse, V.J.; Smith, R.F. (February 1984). Wind Turbine Generator Interaction with Conventional Diesel Generators on Block Island, Rhode Island. Vol. I. Executive Summary. DOE/NASA/0354-1; 47 pp. (NTIS no. DE84015874). Vol. II. Data Analysis. DOE/NASA/0354-2; 138 pp. (NTIS no. DE84016961). Cleveland, OH: NASA Lewis Research Center.

Anderson, T.S.; Bodenschatz, C.A.; Eggers, A.G.; Hughes, P.S.; Lampe, R.F.; Lipner, M.H.; Schomhorst, J.R. (August 1980). MOD-OA 200-kW Wind Turbine Generator Design and Analysis Report. DOE/NASA/0163-2. Cleveland, OH: National Aeronautics and Space Administration; 393 pp. (NTIS no. DE81027625).

\section{Development of vertical-axis systems}

Ashwill, T.D. (July 1992). Measured Data for the Sandia 34-meter Vertical Axis Wind Turbine. SAND-91-2228. Albuquerque, NM: Sandia National Laboratories; 119 pp. (NTIS no. DE92019807).

Clark, R.N. (1991). "Design and Initial Performance of a 500-kW Vertical-axis Wind Turbine." Transactions 34(3); pp. 986-991. New York, NY: American Society of Mechanical Engineers.

Dodd, H.M. (January 1990). "Performance Predictions for an Intermediate-Sized VAWT Based on Performance of the 34-m Vawt Test Bed." Proceedings of the 9th ASME Wind Energy Symposium. Edited by D.E. Berg. SED-Vol. 9. New York, NY: American Society of Mechanical Engineers.

Ashwill, T.D.; Sutherland, H.J; Veers, P.S. (January 1990). "Fatigue Analysis of the Sandia 34-meter Vertical Axis Wind Turbine." Proceedings of the 9th ASME Wind Energy Symposium. Edited by D.E. Berg, SED-Vol. 9. New York, NY: American Society of Mechanical Engineers.

Touryan, K.J.; Strickland, J.H.; Berg, D.E. (November/December 1987). "Electric Power from VerticalAxis Wind Turbines." Journal of Propulsion and Power (3:6); pp. 481-493.

Abramovich, H. (1987). "Vertical Axis Wind Turbines: A Survey and Bibliography." Wind Engineering (11:6); pp. 334-343. Brentwood, England: Multi-Science Publishing Company, Ltd. 
Test and Evaluation of a 500-kW Vertical-Axis Wind Turbine: Final Report. (Southern California Edison Company). (1987). AP-5044. Palo Alto, CA: Electric Power Research Institute; 124 pp.

Berg, D.E. (April 1985). Structural Design of the Sandia 34-m Vertical Axis Wind Turbine. SAND-84-1287. Albuquerque, NM: Sandia National Laboratories; 23 pp.

Nellums, R.O. (February 1985). Field Test Report of the Department of Energy's 100-kW Vertical Axis Wind Turbine. SAND-84-0941. Albuquerque, NM: Sandia National Laboratories; 60 pp.

Design and Fabrication of a Low-Cost Darrieus Vertical Axis Wind Turbine System, Phase II; Volume 1. Executive Summary. (March 1983). SAND-82-7113/1. Albuquerque, NM: Sandia National Laboratories; $51 \mathrm{pp}$.

Design and Fabrication of a Low-Cost Darrieus Vertical Axis Wind Turbine System, Phase II; Volume 2. Final Technical Report. (March 1983). SAND-82-7113/2. Albuquerque, NM: Sandia National Laboratories; $235 \mathrm{pp}$.

Design and Fabrication of a Low-Cost Darrieus Vertical Axis Wind Turbine System, Phase II; Volume 3. Design, Fabrication and Site Drawing. (March 1983). SAND-82-7113/3. Albuquerque, NM: Sandia National Laboratories; $121 \mathrm{pp}$.

Johnston, S.F., ed. (August 1980). Proceedings of the Vertical Axis Wind Turbines (VAWT) Design Technology Seminar for Industry; April 1-3, 1980; Albuquerque, New Mexico. SAND-80-0984. Albuquerque, NM: Sandia National Laboratories; 339 pp.

\section{Innovative wind energy conversion systems}

Noll, R.B.; Ham, N.D.; Azara, J. (Aerospace Systems, Inc.). (September 1985). Flex Rotor Wind Energy Innovative System Assessment. SERI/STR-217-1754. Golden, CO: Solar Energy Research Institute; $118 \mathrm{pp}$.

Walters, R.E.; Migliore, P.G.; Wolfe, W.P. (West Virginia University). (September 1985). Innovative Wind Turbines: The Circulation Control Vertical Axis Wind Turbine. SERI/STR-217-1755. Golden, CO: Solar Energy Research Institute; 81 pp.

Jacobs, E.W.; Lasier, D.D. (January 1984). A Theoretical Analysis of Solar-Driven Natural Convection Energy Conversion Systems. SERI/TR-211-1950. Golden, CO: Solar Energy Research Institute; 37 pp.

Jacobs, E.W. (1983). "Research Results for the Tornado Wind Energy System: Analysis and Conclusions." Solar Engineering-1983: Proceedings of the ASME Solar Energy Division Fifth Annual Conference; April 18-21, 1983; Orlando, Florida. New York, NY: American Society of Mechanical Engineers; pp. 606-617.

South, P.; Mitchell, R.L. (December 1983). Oscillating Wind Energy Conversion Systems. SERI/TR-211-1911. Golden, CO: Solar Energy Research Institute; 25 pp. 


\section{International developments}

National Renewable Energy Laboratory. (1995). International Energy Agency (IEA) Wind Energy Annual Report 1994. NREL/SP-440-7810. Golden, CO: National Renewable Energy Laboratory.

OEM Development Corporation. (March 1993). European Wind Technology. EPRI-TR-101391. Palo Alto, CA: Electric Power Research Institute; 88 pp.

Van Hulle, F.J.L.; Smulders, P.T.; Dragt, J.B. (1992). Wind Energy: Technology and Implementation, Part II. Proceedings of the European Wind Energy Conference, EWEC '91; October 14-18, 1991; Amsterdam, The Netherlands. Amsterdam: Elsevier; 205 pp.

Van Hulle, F.J.L.; Smulders, P.T.; Dragt, J.B. (1991). Wind Energy: Technology and Implementation, Part I. Proceedings of the European Wind Energy Conference, EWEC '91; October 14-18, 1991; Amsterdam, The Netherlands. Amsterdam: Elsevier; 935 pp.

Palz, W. (1990). Proceedings of the European Community Wind Energy Conference; September 10-14, 1990; Madrid, Spain. Bedford, England: H.S. Stephens and Associates; 769 pp.

Ancona, D.F.; Pedersen, B.M.; Pershagen, B. (October 1986). "The International Energy Agency Wind Energy Program." Proceedings of the European Wind Energy Association Conference and Exhibition Vol. 1; October 7-9, 1986; Rome, Italy. Edited by W. Palz and E. Sesto. Rome: A. Raguzzi, Bookshop for Scientific Publications; pp. 129-132.

Selzer, H. (1986). Potential of Wind Energy in the European Community: An Assessment Study. Solar Energy R\&D in the European Community, Series G, Vol. 2. Boston, MA: D. Reidel Publishing Company; 148 pp. 


\section{Index of Authors}

Abramovich, H. (33)

Adler, F.M. (32)

Ancona, D.F. (1), (3), (33), (35)

Anderson, C. (14)

Anderson, T.S. (33)

Araj, K.J. (10)

Ashwill, T.D. (14), (15), (17), (33)

Azara, J. (34)

Babcock, B.A. (13)

Bain, D. (27)

Baker, R.W. (8), (20)

Baker, T.L. (2)

Bankaitis, H. (28)

Barchet, W.R. (7)

Barieau, R.E. (22)

Baring-Gould, E.I. (22)

Barnard, J.C. (10-12)

Barnes, P.R. (21)

Barnwell, R.W. (12)

Barton, R.S. (33)

Berg, D.E. (33), (34)

Bergey, K.H. (5)

Birkenheuer, N.J. (26)

Birn, M.B. (8)

Bodenschatz, C.A. (33)

Bodvarsson, G. (8)

Bollmeier, W.S., II (2)

Britt, J.F. (4)

Brower, M.S. (1)

Buck, J.W. (11), (12)

Buhl, M.L., Jr. (14)

Butterfield, C.P. (12), (14), (17)

Buzenberg, R.J. (22)

Bywaters, G.L. (13)

Cadogan, J.B. (5), (11)

Carlin, P.W. (16)

Carne, T.G. (14)

Cavallo, A.J. (1)

Chan, S.M. (21)

Chang, S.K. (21)

Changery, M.J. (7)

Cheney, M.C. (32)

Chernoff, H. (4), (5)

Childs, S.E. (16)

Chung, S. (10)

Clark, R.N. (15), (20), (22-24), (33)

Coelingh, J.P. (1), (24)

Cohen, J.M. (5)

Coleman, C. (23), (31), (32)

Collins, J.L. (33)
Connell, J.R. (11), (12), (17)

Conopask, H. (4), (5)

Conover, K.E. (13)

Cornaby, B.W. (25)

Corotis, R.B. (9)

Council, C. (8)

Cousineau, K.L. (17)

Cowan, R.A. (23)

Cramer, G. (22)

Curtice, D. (21)

Davey, H.L. (4)

David, M.L. (22)

Davis, R.G. (15), (20)

Dawson, R.E. (23)

Denzler, E.W. (1)

Des Chenes, C. (6), (22)

Desrochers, G. (17)

Divone, L.V. (3)

Dlott, E.H. (21)

Dodd, H.M. (4), (33)

Dodge, D.M. (1-3), (27), (31)

Donovan, J.F. (13)

Doran, J.C. (12)

Dragt, J.B. (35)

Drake, R.L. (8)

Dugundji, J. (10)

Eggers, A.G. (33)

Eggleston, D.M. (3), (10), (13)

Eldridge, F.R. (3)

Elliott, A.S. (14)

Elliott, D.L. (7), (10), (11)

Eppler, R. (15)

Errichello, R. (16)

Fardoun, A.A. (16)

Ferris, J.E. (26)

Fisher, T.A. (10)

Flaim, T. (5)

Fleming, P.D. (2)

Flowers, L.T. (31)

Foote, H.P. (7)

Fraser, D.B. (13)

Freeman, L.N. (14)

Frost, W. (8), (12)

Fuchs, E.F. (16)

Fuchs, J.M. (12)

Garling, W.S. (6)

Garrick, J. (8)

Gaylord, D.R. (9)

George, R.L. (12)

Gilley, J.R. (22) 
Gilmore, E.H. (3), (21)

Gipe, P. (3), (19)

Glynn, E.F. (22)

Gohard, J. (10)

Goldenblatt, M.K. (21)

Golding, E.W. (2)

Goldman, P. (31)

Gower, G.L. (7), (8), (10)

Greeb, F.J. (6), (22)

Grosveld, F.W. (26)

Gyatt, G.W. (12)

Ham, N.D. (13), (34)

Hamrin, J. (27)

Hansen, A.C. (12), (14), (17)

Harper, J. (5)

Haufer, M.R. (6)

Heberling, C.F., III (14)

Hemphill, R.R. (26)

Henry, G. (21)

Herrera, J.I. (16)

Hewson, E.W. (8)

Hiester, T.R. (8), (12)

Hinrickson, E.N. (21)

Hock, S.M. (1), (4), (5), (31)

Hodge, W.T. (7)

Hohmeyer, O. (25)

Holladay, C.G. (7)

Holley, W.E. (13)

Hubbard, H.H. (25), (26)

Hughes, P.S. (16), (23), (31), (33)

Hussaini, M.Y. (12)

Ing, E.T.C. (27)

Jacobs, E.W. (26), (34)

Jacobs, M. (25)

Jager, D. (15)

James, G.H., III (14)

Jamieson, R. (14)

Janetzke, D.C. (13)

Jaras, T. (19)

Jeffries, W.Q. (22)

Johnson, B. (23)

Johnson, B.L., III (23)

Johnson, G.L. (2), (22)

Kaplan, M.M. (1)

Karas, K.C. (4)

Kaupent, B.M. (21)

Kelley, N.D. (10), (11), (15), (17), (26)

King, W.R. (23)

Kleinkauf, W. (22)

Klimas, P.C. (15)

Knowles, R. (4), (5)

Kocurek, D. (15)

Koeppl, G.W. (2)

Korn, D.H. (21)
Kronenburg, J.C. (10)

Kruse, V.J. (23), (33)

Kurtz, E.B. (28)

Lamarre, L. (1)

Lampe, R.F. (33)

Lasier, D.D. (34)

Lauffer, J.P. (14)

Lauw, H.K. (16)

Lawler, J.S. (21)

Lawless-Butterfield, C. (27)

Lawrence, K.A. (25)

Laxson, A.S. (31)

Le Gourieres, D. (3)

Leonard, T.M. (15)

Lipner, M.H. (33)

Lissaman, P.B.S. (12)

Liu, H.-T. (11)

Lobitz, D.W. (14)

Long, B.H. (12)

Loose, R. (31)

Lornell, R. (27)

Lundsager (23)

Lynch, J. (31), (32)

Mandell, J.F. (17)

Manwell, J.F. (13), (22), (23)

Marah, F. (21)

Marckx, D.A. (16)

Marjan, P.L. (22)

Marrs, R.W. (9)

Martinez-Sanchez, M. (10)

Masse, B. (17)

Matson, D.F. (12)

Maule, P.A. (8)

Mayer, D.J. (31), (32)

McCabe, T. (21)

McCarroll, D.A. (4)

McCarthy, E.F. (7)

McClenahan, M.R. (8)

McGowan, J.G. (23)

McKenna, H.E. (26)

McNerney, G.M. (12)

McNiff, B.P. (14), (16)

Merrill, O. (4), (5)

Migliore, P.G. (34)

Miley, S.J. (15)

Miller, A.H. (4), (8), (10)

Miller, L.S. (16)

Milligan, M.R. (4)

Mitchell, R.L. (34)

Moment, R.L. (5), (19)

Moretti, P.M. (3)

Morris, V.R. (10), (11), (17)

Moyle, R.A. (4)

Mulh, K.E. (22) 
Musial, W.P. (12), (16)

Nagle, R. (5)

Nellums, R.O. (34)

Nelson, V. (3), (21), (22)

Niak, D. (15)

Nierenberg, R. (11)

Nolan, P.J. (21)

Noll, R.B. (13), (34)

Norgaard (23)

Norton, J.H., Jr. (32)

Oberg, K.W. (27)

Orgill, M.M. (8)

Ostowari, C. (15)

Packard, K.R. (12)

Palz, W. (35)

Park, J. (3), (19)

Pastorel, H. (17)

Patton, J.B. (4)

Pedersen, B.M. (35)

Pennell, W.T. (8)

Percival, D. (5)

Pershagen, B. (35)

Phillips, P.D. (27)

Poor, R.H. (33)

Porter, B.K. (13)

Powell, D.C. (11)

Probert, S.D. (2)

Quinlan, P. (8)

Rader, N. (27)

Ramsdell, J.V. (7)

Reddoch, T.W. (16)

Redman, C.W. (25)

Redmond, I. (14)

Reed, R.M. (17)

Renne, D.S. (12)

Rodgers, J. (31)

Rogers, S.E. (25)

Rohrbach, C. (13)

Rosenfeld, C.L. (8)

Saeed, A. (16)

Samborsky, D.D. (17)

Sandusky, W.F. (7)

Schaller, D.A. (27)

Schluter, L.L. (16)

Schneider, A.D. (22)

Schornhorst, J.R. (33)

Schott, T. (22)

Schwartz, M.N. (7)

Schweizer, T.C. (4), (5)

Schwind, D. (3)

Scott, G.N. (12)

Scott, G.W. (23), (33)

Selig, M.S. (13)

Selzer, H. (35)
Sengupta, D.L. (26)

Senior, T.B. (26)

Shaltens, R.K. (33)

Shepherd, D.G. (2)

Shepherd, K.P. (25), (26)

Sherwin, R. (23)

Shieh, C.F. (8)

Shoemaker, T.M. (28)

Shultis, J.K. (22)

Simms, D.A. (12), (17)

Simon, R.L. (11), (12)

Skroski, J.C. (21)

Smith, B. (15), (17)

Smith, C.E. (14)

Smith, D.R. (1)

Smith, J.C. (23)

Smith, M.F. (6), (22)

Smith, R.F. (23), (33)

Smulders, P.T. (35)

Snow, A.L. (14)

Soderholm, L.H. (22)

Somers, D.M. (15)

South, P. (34)

Spera, D.A. (3), (10), (19), (33)

Stafford, R.W. (6), (22)

Starcher, K. (3), (21)

Stein, W.M. (22), (23)

Stephens, D.G. (26)

Stern, W. (23)

Sticksel, P.R. (25)

Stiller, P.H. (23), (33)

Stoddard, F.S. (3), (10), (13), (15)

Strickland, J.H. (33)

Strojan, C.L. (25)

Sullivan, R.L. (5)

Sutherland, H.J. (17)

Swift, A. (4), (31)

Swisher, R. (1)

Talle, D.A. (25)

Tangler, J.L. (15), (17)

Taylor, R.B. (32)

Tennis, M.W. (1), (21)

Thresher, R.W. (1), (3-5), (13), (14), (31)

Tomich, S.D. (10)

Torrey, D.A. (16)

Torrey, V. (2)

Touryan, K.J. (33)

Turner, R.E. (12)

Vachon, W.A. (16)

Van Bibber, L.E. (14)

Van Hulle, F.J.L. (35)

Van Wijk, A.J.M. (1), (24)

Veers, P.S. (11), (17), (33)

Viterna, L.A. (13) 
Wade, J.E. (8), (13), (20)

Wagner, J.P. (22)

Wainauski, H. (13)

Waldon, C.A. (17)

Walker, S.N. (8), (13), (14), (20)

Walters, R.E. (34)

Weaver, N.L. (6), (22)

Weber, T.L. (14)

Wegley, H.L. (8), (11), (12), (21)

Wehrey, M. (14)

Weigand, C.H. (16)

Welch, M. (6)

Wendell, J. (10)

Wendell, L.L. (7), (8), (10)

Whalen, W. (25)

Williams, T. (1)

Wilreker, V.F. (23), (33)

Wilson, R.E. (13), (14)

Wolfe, W.P. (34)

Woolley, S.G. (8)

Worabel, R. (13)

Wright, A.D. (10), (13), (14)

Wschenbach, W. (22)

Wyatt, A. (5), (21), (24)

Wysocki, K.J. (4)

Yekutieli, O. (15)

Younkins, T.D. (20)

Zalay, A.D. (12)

Zickefoose, C.R. (32) 


\section{Index of Companies and Organizations}

Acoustic Society of America (25)

Aerospace Systems, Inc. (13), (34)

Agricultural Research Service (22)

Altamont Energy Corporation (11)

Alternative Energy Institute (3), (21)

American Society of Mechanical Engineers (3), (5),

(8), (10-19), (21), (22), (23), (29), (31), (33), (34)

American Solar Energy Society (1), (19)

American Wind Energy Association (1-5), (7), (8),

(10), (13), (14), (15-25), (27-29), (31)

Atlantic Orient Corporation (16), (23), (31)

Battelle Columbus Laboratory (25)

Bergey Windpower Company, Inc. (5)

Bonneville Power Administration (27)

Bureau of Reclamation (21)

California Energy Commission (1), (7), (20), (25)

Canadian Wind Energy Association (1), (31)

Colorado State University (7), (17)

Computational Methodology Associates (15)

Cornell University (2)

Decision Focus, Inc. (20)

Electric Power Research Institute (1), (4), (5), (7), (8), (16), (19), (20); (21), (24), (25), (34), (35)

Electronic Power Conditioning, Inc. (16)

Electrotek Concepts, Inc. (16), (20), (23)

Enertech Corporation (32)

ESI Energy, Inc. (27)

Fayette Manufacturing Corporation (11), (12)

FloWind Corporation (11), (31)

FWG Associates, Inc. (8)

GEARTECH (16)

General Electric Company (19)

Grumman Energy Systems, Inc. (32)

Hamilton Standard (13)

International Electrotechnical Commission (18), (28), (30)

International Energy Agency (1), (4), (17), (18), (21), (25), (26), (29), (35)

JBF Scientific Corporation (5)

Kenetech (7), (25)

Litchfield Consulting Group (20)

Lockheed-California Company (19)

Massachusetts Department of Public Utilities (25)

Montana State University (17)

NASA Langley Research Center (26)

NASA Lewis Research Center (13), (23), (28), (32), (33)

National Aeronautics and Space Administration (12), (33)

National Climatic Data Center (7)
National Fire Protection Association (28)

National Renewable Energy Laboratory (1), (2), (4), (5), (7), (10-17), (20), (25), (31), (33), (35)

National Research Council (10)

NEOS Corporation (8)

Nielsen Engineering and Research, Inc. (3)

Niles \& Associates, Inc. (22)

North Wind Power Company, Inc. (31), (32)

Northern Power Systems (13), (23), (31)

Northwestern University (9)

Oak Ridge National Laboratory (20)

OEM Development Corporation (24), (35)

OMNION Power Engineering Corporation (16)

Oregon State Department of Energy (27)

Oregon State University (8), (14), (20)

Pacific Gas and Electric Company (20)

Pacific Northwest Laboratory (7-9), (11), (12), (17),

(21)

Pacific Wind Energy (8), (13)

Peace Corps (22)

R. Lynette \& Associates, Inc. (13), (20), (31)

Rensselaer Polytechnic Institute (16)

Research Triangle Institute (5), (21), (24)

Riso National Laboratory (23)

Rockwell International Corporation (5), (19), (32)

Rocky Flats Wind Energy Research Center (13), (15),

(17), (27), (32)

Sandia National Laboratories (11), (14-17), (19), (33),

(34)

Science Applications International Corporation (5),

(23)

Second Wind Inc. (16)

Solar Energy Industries Association (19)

Solar Energy Research Institute (4), (5), (8), (11), (12-17), (23), (25-27), (31), (32), (34)

Southern California Edison Company (34)

Stadia, Inc. (19)

Stanford University (17)

Strategies Unlimited (19)

Texas A\&M University (15)

U.S. Department of Agriculture (22)

U.S. Department of Energy (1), (2), (6), (8), (10), (13), (16), (20-22), (27)

U.S. Department of the Interior (21)

U.S. Windpower, Inc. (7), (12)

Union of Concerned Scientists (1)

United Technologies Research Center (32)

University of Colorado (16)

University of Massachusetts (13), (22)

University of Michigan (26) 
University of Texas (4), (31)

University of Utah (14)

University of Washington (16)

University of Wyoming (9)

Utility Wind Interest Group (4), (7), (20), (25)

Utrecht University (1), (24)

W.A. Vachon \& Associates (16)

Washington State Energy Office (27)

West Virginia University (34)

Western Area Power Administration (8)

Westinghouse Electric Corporation (14)

Zaininger Engineering Company, Inc. (17)

Zond Systems, Inc. (17) 


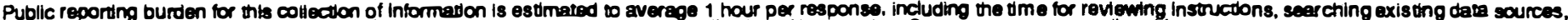

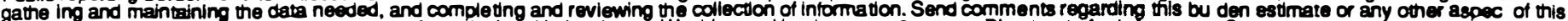

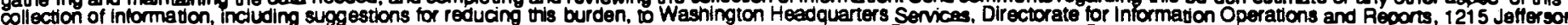
Davis Highway, Sulte 1204, Arlington, VA 22202-4302, and to the Offlce of Manegement and Budget, Paperwork Reducton Proiect (0704-0183), Washington. DC 20503.

1

2. REPORT DATE February 1995
3. REPORT TYPE AND DATES COVERED Spocial publication
4. TITLE AND SUBTITLE

Wind Energy Bibliography

6. AUTHOA(S)

7. PERFORMING ORGANIZATION NAME(S) AND ADDRESS(ES)

\section{FUNDING NUMBERS}

C:

TA: WE517110

8. PERFORMING ORGMNIZATION REPORT NUMBER

\section{SPONSORINGMONITORING AGENCY NAME(S) AND ADDRESS(ES)}

National Renewable Energy Laboratory

1617 Cole Bivd.

Golden, CO $80401-3393$
10. SPONSORINGMONITORING AGENCY REPORT NUMBER

SP-440-6642

DE94006896

11. SUPPLEMENTARY NOTES

12a. DISTRIBUTION/AVAILABUITY STATEMENT National Technical Information Service U.S. Department of Commerce 5285 Port Royal Road Springtield, VA 22161 12b. DISTRIBUTION CODE

UC-1210

13. ABSTRACT (Maximum 200 words)

This bibliography is designed to help the reader search for information on wind energy. The bibliography is intended to help several audiences, including engineers and scientists who may be unfamiliar with a particular aspect of wind energy, university researchers who are interested in this field, manufacturers who want to learn more about specific wind topics, and libranians who provide information to their clients. Topics range from the history of wind energy use to advanced wind turbine design. In addition to wind turbine development, design and applications, references for wind energy economics, the wind energy resource, and environmental and institutional issues related to wind energy are also included. This document was prepared by the National Renewable Energy Laboratory in support of the U.S. Department of Energy under contract number DE-AC36-83CH10093.

14. SUBJECT TERMS

15. NUMBER OF PAGES

renewable energy; wind energy; wind turtine design; wind turbines-electricity generation; wind energy systems development; wind turbine development; wind turbine testing; U.S. Department of Energy Wind Program; wind energy conversion systems; wind energy—design, utility applications, residential applications, international applications, utility integration, economics, economic analysis, environmental issues, avian issues, insottutional issues, resource assessment

17. SECURITY CLASSIFICATION OF REPORT Unclassified
18. SECURITY CLASSIFICATION OF THIS PAGE Unclassified
19. SECURITY CLASSIFICATION OF ABSTRACT Unclassified
16. PRICE CODE

20. LIMITATION OF ABSTRACT

UL

NSN 7540-01-280-5500

Standard Form 298 (Rov. 2-89) 